\title{
Research Square \\ Cooperative Fusion Architecture-based Distributed Spectrum Sensing Under Rayleigh Fading Channel
}

\section{Rania A. Mokhtar}

Taif University

Rashid Saeed ( $\nabla$ eng_rashid@hotmail.com )

Taif University https://orcid.org/0000-0002-9872-081X

Hesham Alhumyani

Taif University

\section{Research Article}

Keywords: Cognitive radio network (CRN), Spectrum sensing, Cooperative, Distributed architecture, Decision fusion

Posted Date: March 15th, 2021

DOl: https://doi.org/10.21203/rs.3.rs-284176/v1

License: (c) (i) This work is licensed under a Creative Commons Attribution 4.0 International License. Read Full License

Version of Record: A version of this preprint was published at Wireless Personal Communications on January 30th, 2022. See the published version at https://doi.org/10.1007/s11277-021-09386-z. 


\title{
Cooperative Fusion Architecture-based Distributed Spectrum Sensing under Rayleigh Fading Channel
}

\author{
Rania A. Mokhtar ${ }^{1}$, Rashid A. Saeed ${ }^{1}$, and Hesham Alhumyani \\ ${ }^{1}$ Deparment of Computer Engineering, Taif University, Taif 21944. Saudi Arabia \\ Corresponding author: Rania A. Mokhtar (e-mail: ramohammed@tu.edu.sa).
}

\begin{abstract}
Cognitive radio (CR) is one of the most promising technology soon due to the scarcity of the spectrum, especially at microwave band. CR faces massive resistance from the industry because of the potential interference caused by the secondary users. Spectrum sensing forms an important functionality for CR systems. However, such detection performance is usually compromised by shadowing and fading channel conditions. Cooperative sensing is one of the crucial solutions to overcome degraded detection performance. To improve the sensing performance and reduce the reporting error, a distributed architecture for processing and fusion of sensing information is proposed in this work. In dense network scenarios, the decision fusion for cooperated users could be complex and reported sensing traffic may require large bandwidth. This paper proposes a new distributed detection and adapted threshold based on controlled false alarm probability to improve sensing reliability and efficiency in a highly Rayleigh faded environment. A distributed detection is developed by selecting fusion nodes (FN) that are dynamically selected from a group of nodes. The detection threshold is calculated adaptively using the link quality indicator (LQI) of the sensing channel. Moreover, the proposed method can significantly minimize the typically transmitted bits in the reporting channel. The paper also discussed in detail the design parameter of the CR number on the performance of fusion values. The simulation analysis shows that the performance of the distributed cooperative sensing (DCS) process is considerably improved by the adapted threshold. The numerical results demonstrated that the error was remarkably minimized. The ROC curve of the sensing process is notably improved for detection probability and false alarm probability, respectively. Finally, it was shown that the requirement of sensitivity can be greatly improved up to 0.95 .
\end{abstract}


INDEX TERMS Cognitive radio network (CRN); Spectrum sensing; Cooperative; Distributed architecture, Decision fusion

\section{INTRODUCTION}

Radio spectrum becomes one of the international wealth that get the attention of researchers and academia since early this century. Spectrum bands especially those between $300 \mathrm{MHz}$ to $10 \mathrm{GHz}$ become quite crowded with many wireless technologies, services, and devices. The wireless industry and vendors are looking eagerly for new opportunities for the highly demanded wireless communication market. The industry now looking for more freely spectrum or unutilized spectrum such as $700 \mathrm{MHz}$ to be occupied for broadband services where it has been auctioned in many countries. for the sake of new spectrum, some new technologies recently have been innovated at terahertz (THz) and visible light (VL) bands. The FCC report [1] indicates that: some frequency bands in the spectrum are largely unoccupied most of the time, some other frequency bands are only partially occupied, and the remaining frequency bands are heavily used. Cognitive Radio (CR) is currently considered as one of the most promising solutions to the aforementioned spectrum scarcity problem and efficiently usage of many underutilization licensed frequency bands [2, 3].

Cooperative Cognitive Radio has been introduced to improve the spectrum sharing efficiency, improve the capacity and avoid interference between various radios that work in the same frequency [4-7]. Wireless communications have many impairments, problematic issues, and challenges; in the cognitive radio arena, these issues become severe and critical due to collisions and conflict that may happen in the radio access network (RAN). Hidden node problem and synchronization are an example, in addition to many problems that Rayleigh fading may cause. Therefore, one of the key functions that CR needs to be aware of is detection reliability. Many research and studies have been proposed to increase the detection and sensing reliability to ensure no disturbance can be caused due to coexistence and spectrum sharing. in fact, the industry could not reach a consensus for cognitive radio standards that due to the high probability of disturbance for the primary users [8].

The cognitive radio may not be able to reliably detect a Primary User (PU), this is due to the signalto-noise ratio (SNR) of the PU signal can be extremely low due to Rayleigh fading and shadowing. Weak SNR with high probability can lead to hidden node problem, which it can cause severe interference problem to the PUs [9-12]. Secondary Users (Sus) may be hidden by a shadow from the PU transmitter however in the meantime, PUs receiver may be close enough to the SU that is not hidden by a shadow from the PU transmitter. Therefore, may start sending although the PU is using the same frequency, and hence interfering with the intended receiver of the transmission. Also, [13] 
shown that when the number of cognitive users increases the probability of bit error is also increased, which dramatically reduces the spectrum agility.

Due to all these problematic issues, cooperative spectrum sensing in cognitive radio has been proposed recently to support the decision of the local sensing /detection and improve the probabilities of false alarm and detection [14-17]. Rayleigh fading channel is the main cause of low SNR in practical, so many related works have been suggested for the obligation of users' cooperation to cope with the problem of cognitive radio detection. Cooperative sensing achieves diversity gain and can reduce the detection time and thus increase the overall spectrum agility [18]. However, the cooperative sensing performance can be severely degraded due to fading reporting/control channel [19], where usually the low SNR is used. Also, with a large number of cognitive users, the decision fusion for cooperated users could be complex and the reporting sensing result may require a large bandwidth [20].

A hard decision auto-correction reporting system is proposed in [21]. Where an auto-correction scheme is introduced for the reported bit error, and then reduces and minimizes the number of bits in the reporting channels by letting the user with detected information only to send its report. Even though this method reduced the required bandwidth and energy in the reporting channel, however, due to only a few SUs are reporting their observations without hearing from the other users, the spectrum agility can be degraded dramatically with the number of users in the network.

One possible approach to increase the spectral agility and decrease the probability of interference of cognitive radios to the existing radio systems is proposed by [23] in which distributed spectrum sensing has been used. In [24] a cluster-based cooperative spectrum sensing method is proposed, by separating all the secondary users into a few clusters and selecting the most favorable user in each cluster. Another kind of cooperative spectrum sensing was proposed in [25], [26], and [27], where a power-constrained cooperation scheme is discussed. In which the cognitive user near the PU is regarded as a relay to forward its sensing data to the one who receives a weak signal from the PU due to fading and shadowing. However, when the number of cognitive users increases this requires a high bandwidth reporting channel, and the probability of error becomes higher, which will lead to wrong decision at the fusion point.

The rest of this paper is organized as follows: Section 2 is presenting the system model of the local sensing based on the energy detection and network architecture based on the distributed cooperative sensing (DCS) and cooperation behaviors of the system Section 3 shows the analytical and simulation results. Finally, section 4 concludes the paper.

\section{MAIN AIM AND CONTRIBUTION}


In this paper, cooperative cognitive radio detection has been introduced to utilize spatial and temporal diversity for multiuser detection reliability [28-33]. Cooperative spectrum sensing is proposed to overcome the fading, shadowing, and noise uncertainty problems in a channel. Cooperating is quite successful in dense urban and urban scenarios due to the number of participated nodes in the cooperative process. Cooperative also can work for wide coverage areas where it offers a reliable answer to the hidden-terminal issue since SUs are apart by a larger distance than the correlated (shadow, fading) distance which this distance makes it unlikely for two SUs to be shadowed instantaneously from the PUs. related works on cooperative cognitive radio sensing among users [39, 40] have investigated methods where cooperative detection has exploited all users in the cognitive radio network $(\mathrm{CRN})$. Literature proofed that this scheme vitally enhanced the detection reliability of PU activity. The problem statement we are addressing in this paper as follows:

- Unreliable ED (energy detection) monitoring in local users sensing addition to deficient (faded) reported detection through reporting channel with limited bandwidth under the hypothesis of a large number with long distances of cognitive nodes

- Decision fusion procedure complexity; fusion is a centralized-based idea that has many problematic issues such as computation complexity, single point of failure, and energy consumption.

In this paper, we propose a distributed sensing where the Cognitive Radios (CR) are divided into subgroups. The users perform sensing using energy detection (ED) and then report their observations using auto-correction based on the best SNR reporting channel. The processing and fusion of spectrum observations are done by each group by electing one fusion node (FN); fusion node is selected based on the best signal-to-noise ratio which we will express here with $\gamma$. the procedure would be like follows:

- The group is collaboratively making the sensing decision,

- fusion center (FC) is received the group decision by using the reporting channel.

- The fusion center (FC) produces the final decision and final spectrum usage map

Furthermore, an adaptive medium access control protocol is proposed for data exchange where the noise estimation is also used for the nodes reporting scheduling; the proposed scheme increases the time of sensing for the delayed scheduled CR nodes. The paper proposed distributed cooperative sensing (DCS) technique with overcoming of low SNR causes by the Rayleigh fading report channel, which shows a great enhancement in detection readability. in the simulation results and numerical analysis. The detection probability, false alarm probability and reported bits error rate were examined in the proposed model as performance metrics to validate and verify the proposed distributed decision fusion architecture for the cooperative spectrum sensing for direct cognitive radio-distributed decision 
fusion architecture under Rayleigh fading channel and non-cooperative detection. Also, an investigation and optimization of the distributed cooperative gain, an analytical formulation with probable candidate node selection criteria are used.

\section{SYSTEM MODEL}

In the CR network, in order to recognize white space channels that are available for transmission, the CR nodes accomplish active detection of the surrounding through (passive) for frequency time, and space degrees of freedom [41]. Then. nodes send their local sensing -under a maintained probability of false alarm- to fusion node (FN) user. The fusion user makes the decision of the group and forwards the reporting bits to the fusion center (FC). The fusion center produces the final decision and final spectrum usage map. The network scenario is shown in Figure 1. An AWGN channel with squared power path loss is assumed for CR-to-CR \& CR-to-FN transmission.

Assuming communication from FN-to-FC faded by Rayleigh characteristics. Moreover, in the network for the cooperative nodes, the distance between any two nodes compared with the wavelength leads to independent fading coefficients [43]. The rationale behind such channel assumptions is that the fusion node (FN)-to-fusion center transmission distance is much larger than the CR-to-FN and FNto-FN transmission range and the communication environments are more complex.

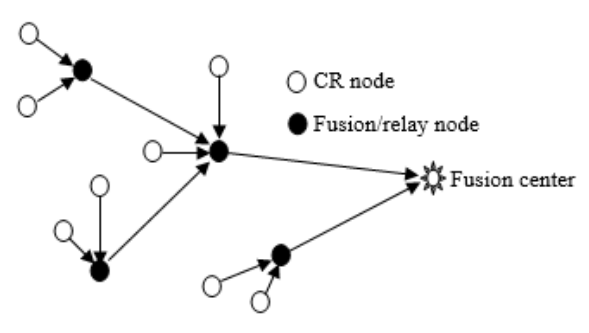

FIGURE 1: Network Architecture

\section{A. Distributed Spectrum Sensing}

The distributed cooperation architecture is known to have the potential to increase spectral estimation reliability and decrease the probability of interference of cognitive radios to existing radio systems. The Network architecture consists of two basic elements: the sensing group and the Sensing Coordinator (SC) [44-46]. The sensing group is divided into G sensing subgroups which each subgroup performed by $\mathrm{n}$ secondary users.

The secondary cognitive radio operates in a distributed cooperative group manner, where the fusion processing of local spectrum sensing for each group [47]. The detection decision at the group level is made to be sent through the best SNR reporting channel. These processes are spanning through both PHY and MAC layers, where the spectrum sensing and signal detections are performed by using PHY 
parameters. While choosing the group head GH is done through both PHY and MAC, which are based on the best SNR, the groups elect their GH using MAC messages. This can be performed by using a self-organizing algorithm.

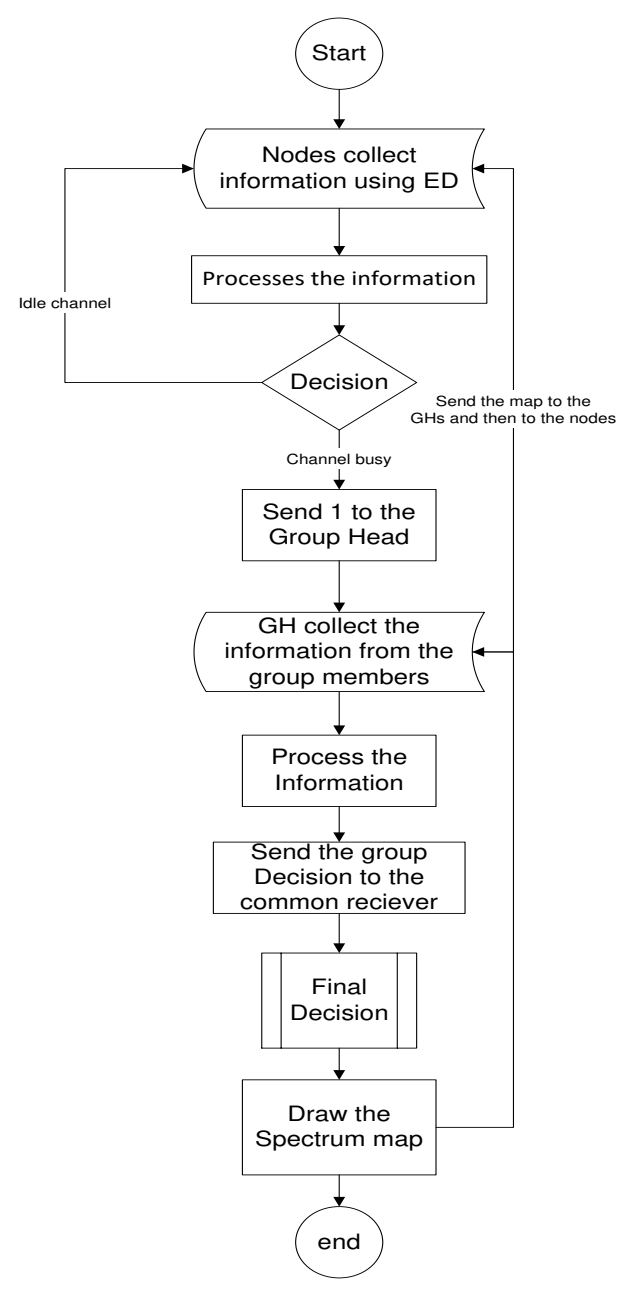

\section{FIGURE 2: The decision stages for the distributed cooperative spectrum sensing}

The GH communicates the SC (i.e., a base station (BS)) through the best SNR. The BS and uses the Radio Resources Management (RRM) feature in the MAC layer to make the spectrum decisions. The flow chart of the decision stages is described in Figure 2. Algorithm 1 shows the details of the distributed cooperative sensing (DCS) pseudo-code. Figure 3 shows the Distributed sensing stages.

Assume that all the channels to be experienced Rayleigh fading. Moreover, channels corresponding to different cognitive users are assumed to be independent. Thereby, the signal received at the SU's antenna can be expressed as follows [48]: 


$$
s[n]=h x[n]+w[n]
$$

where $w[n]$ is the noise which is here we consider it as AWGN, $x[n]$ is the detected signal, $n$ is an indication of number of samples for the discrete channel index, and $h$ is the impulse of fading channel. Here we considered that $x[n]=0$ in the case that there are no PU signals [49]. The decision on the presence of a primary user signal can be made by comparing the decision metric $M$ against a fixed threshold.

\section{Algorithm 1: The proposed distributed cooperative sensing (DCS) pseudo code}

Apply distributed architecture in CRN

apply k-mean for node grouping

give ID (identification) for all groups

For all groups select best reporting channel associated with a node and assign as

fusion/relay node

compute gain of channels at fusion center

announce the fusion/relay nodes for each group

For all groups define the threshold for PU detection

calculate the noise at fusion/relay node

compute the $\mathrm{P}_{-} \mathrm{f}$

$\%$ noise calculation is out of this work scope, so is selected as $P \_f=0.1$

Compute the threshold for $P_{-} f=0.1$

Apply ED in each group's node given the calculated threshold

$P$ _received $\leq$ threshold

send this result to fusion/relay node

run the fusion/relay node decision procedure

Run $O R \_r u l e$ for received reports

Send the decision to fusion center

Run the final decision procedure

Use $O R \_r u l e$ for the received fusion decisions

Assess the parameters for system performance

Evaluate ROC parameter

$\%$ simulate,

Save results history and evaluated with the on/off mode

Calculate probability of error for reporting channel

Calculate sensitivity level 
Use node_selection procedure to decrease the bandwidth

Let nodes with detection information to send their decision

Modify any no_detection status to detection

Re-calculate the parameters for system performance

Store all evaluated performance parameters and bandwidth value

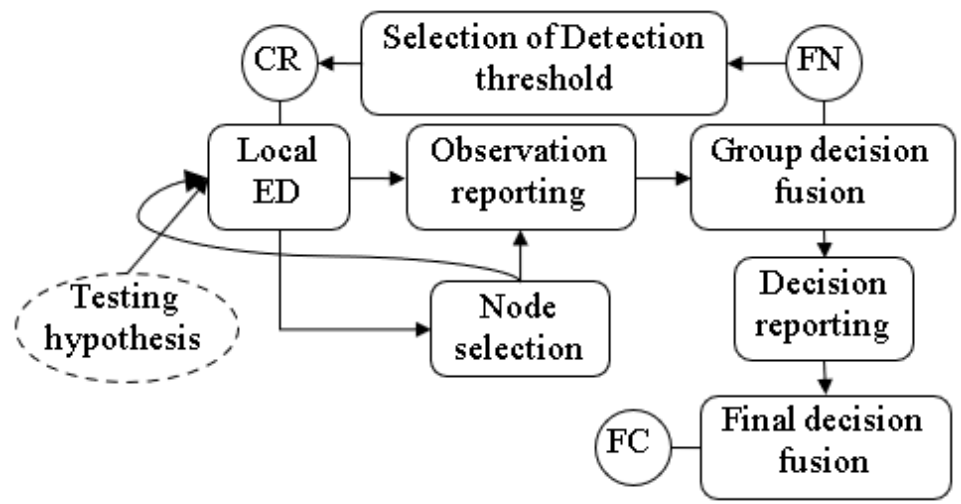

Figure 3: Distributed sensing stages

This is equivalent to distinguishing between the hypothesis testing busy channel, $H_{1}$, and idle channel, $H_{0}$, which can be expressed by [50]:

$$
\begin{array}{cc} 
& H_{0}: \quad s[n]=w[n] \\
H_{1}: & s[n]=h x[n]+w[n]
\end{array}
$$

Energy detection (ED) is one of most techniques used in detection and estimation of spectrum sensing for unknown signals. The detection of unknown signals using ED the mathematical expression can be modeled as [51],

$$
M=\sum_{n=0}^{2 N-1} s[n]
$$

The additive white Gaussian noise is designed analytical as a normal distribution random variable where the mean $\mu=0$ where the variance is $\sigma_{w}^{2}$ i.e.

$$
\begin{array}{r}
w[n]=N\left(0, \sigma_{n}^{2}\right) \\
w[n]=\frac{1}{\sigma_{w} \sqrt{2 \pi}} e^{-\frac{1}{2}\left(\frac{n}{\sigma_{w}}\right)^{2}}
\end{array}
$$


The decision statistics $\mathrm{I}$ is a probability density function which assumed to be chi-square distribution, $\chi^{2}$, with degrees freedom $k=2 N$, in case of true hypothesis $H_{0}$; else, would be as noncentral with (mean $\mu=0$ ) chi-square distribution $\chi^{2}$. Which can be expressed as:

$$
M \sim\left\{\begin{array}{cc}
\chi_{2 N}^{2} & H_{0} \\
\chi_{2 N\left(\gamma_{i}\right)}^{2} & H_{1}
\end{array}\right.
$$

If we assumed that the sample is quite large then the distribution can be approximated as central limit theorem with normal Gaussian distribution using the, so equation 5 of the Chi-distribution $M$ can be rewritten in the following format [54],

$$
\begin{array}{r}
M \mid H_{0} \approx N\left(N \sigma_{x}^{2}, 2 N \sigma_{w}^{2}\right) \\
M \mid H_{1} \approx N\left(N\left(\sigma_{x}^{2}+\sigma_{w}^{2}\right), 2 N\left(2 \sigma_{x}^{2}+\sigma_{w}^{2}\right)\right)
\end{array}
$$

where $N\left(\sigma_{x}^{2}+\sigma_{w}^{2}\right)$ known as central limit theorem with normal Gaussian distribution with mean $\sigma_{x}^{2}$ and variance $b$. The detection threshold $\lambda$ for a given $P_{f}$ can be estimated as [55]:

$$
\lambda=\sqrt{2 N \sigma_{x}^{2}} Q^{-1}\left(P_{f}\right)+N \sigma_{w}^{2}
$$

where $Q(a)=\int_{a}^{\infty} \frac{1}{\sqrt{2 \pi}} \ell^{\frac{a^{2}}{2}} d a$

Undertake that $k$ cognitive users are contained in a group of $G_{j}$ where $\left(K \in K_{t}\right)$, where $K_{t}$ is the cognitive user's number in the whole network which contains a certain number of groups. So, the decision statistics $M$ for group $M_{j}$ that collaboratively sense the PUs can be expressed as

$$
M_{j}=\sum_{K=0}^{K-1} \sum_{n=0}^{2 N-1} s_{j}[n]
$$

The distribution probability of the cooperative sensing group $M_{j}$ approximated as the central limit theorem with chi-square random distribution. The decision statistics $M$ for group $M_{j}$ that collaboratively sense the PUs can be expressed:

$$
M_{j} \mid H_{1} \sim N\left[N\left(\sum_{K=0}^{K-1} \sigma_{x, j}^{2}+J \sigma_{w, j}^{2}\right),\right.
$$




$$
\begin{gathered}
\left.2 N\left(2 \sum_{K=1}^{K-1} \sigma_{x, j}^{2}+J \sigma_{w, j}^{2}\right)\right] \\
M_{j} \mid H_{0} \sim N\left(N K \sigma_{x}^{2}, 2 N K \sigma_{w}^{2}\right)
\end{gathered}
$$

then the detection threshold $\lambda_{j}$ for a given $P_{f}$ can be estimated as:

$$
\lambda_{j}=\sqrt{2 N K \sigma_{x}^{2}} Q^{-1}\left(P_{f}\right)+N K \sigma_{w}^{2}
$$

From (7) and (8) and following same derivation in [57-59], then the probability of detection $P_{d}$ for the local nodes and sensing group can be written for $\mathrm{k}$ total number cognitive users and $j$ total number of sensing groups as:

$$
\begin{gathered}
P_{d, k}=Q\left(\frac{\lambda-N\left(\sigma_{x}^{2}+\sigma_{w}^{2}\right)}{\sqrt{2 N\left(2 \sigma_{x}^{2}+\sigma_{w}^{2}\right)}}\right) \\
P_{d, j}=Q\left(\frac{\lambda_{j}-\left(N\left(\sum_{K=0}^{K-1} \sigma_{x, j}^{2}+K \sigma_{w, j}^{2}\right)\right)}{\sqrt{2 N\left(2 \sum_{K=0}^{K-1} \sigma_{x, j}^{2}+K \sigma_{w, j}^{2}\right)}}\right)
\end{gathered}
$$

\section{B. Auto-correction}

In the cooperative sensing and the ED level, the secondary users (SUs) have to decide that if the primary users (PUs) signal is detected or not [60]. For that, the integrator output $Q$ is associated with the threshold $\lambda$. If the value of $Q$ is exceeded $\lambda_{2}$ the threshold 2, a report of the decision $H_{1}$ is taken and decision of binary 1 is sent to group head GH and if $Q$ lower than $\lambda_{2}$, the threshold 2 a report the decision $H_{0}$ is also taken and the decision of binary 01 is sent to fusion node (FN) else "no decision" is considered. This concept can be summarized and calculated as:

$$
\left\{\begin{array}{cc}
H_{0} & Q<\lambda_{1} \\
H_{1} & Q>\lambda_{2} \\
\text { nodecision } & \lambda_{2}>Q>\lambda_{1}
\end{array}\right.
$$

Equation (11) is illustrated in Figure 4(a). To reduce the number of reporter bits, in this paper we proposed a single reporting bit using auto-correction scheme, where only one threshold $\lambda$ is introduced. If the value of $Q$ is exceeded $\lambda$, a report of the decision $R$ is considered and the decision of binary 1 is sent to Fusion nodes (FNs) otherwise "no decision" decision $R^{\prime}$ is taken. This is given by: 


$$
R=\left\{\begin{array}{cc}
1 & Q>\lambda \\
\text { nodecision } & Q<\lambda
\end{array}\right.
$$

This is can be illustrated as shown in Figure 4(b).

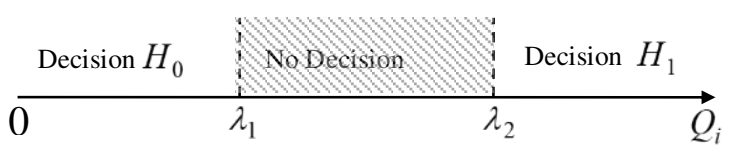

(a)

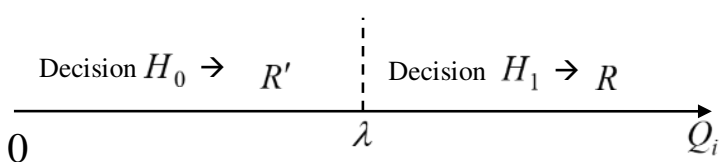

(b

FIGURE 4: (a) Cooperative sensing detection technique with two thresholds for local user. (b) Auto-correction technique with single threshold.

Assume that the group head GH receiver receive $k$ (out of $K$, where $K$ is the is the cognitive user's number in the whole network, while $\mathrm{k}$ is the number of users per group) local decision by the cognitive cooperative users. If the $\mathrm{FN}$ receives locally the decision 0 instead of 1, it's taken as a report of error due to the faded and attenuated channel and the decision to be auto-corrected to the decision 1 [64].

Under these circumstances the performance will be high regardless of the channel condition. The ultimate sensing decision $H$ is taken based on the coefficient $k^{\prime}$ at the FN server If the FN server obtains any local sensing decision 0 or 1 a final decision $\mathrm{H}=1$ is considered. If no local sensing decision is received to the $\mathrm{FN}$ server, then a final sensing decision $\mathrm{H}=0$ is considered. This is can be illustrated as:

$$
H= \begin{cases}1 & k^{\prime} \text { localdecisions } k \leq K \\ 0 & \text { nolocaldecision } \quad k=0\end{cases}
$$

Let $\bar{k}$ denote as normalize mean number of bits reporting; then:

$$
\bar{k}=k_{a v g} / K
$$

$k_{a v g}$ denotes as mean bits number in reporting stage. assume that $R_{k}$ is the state of $k$ reporting from users, and $R_{K-k}^{\prime}$ is the state of $K$ - $k$ not reporting of cognitive users, from (12) we can write:

$$
P\left\{R_{k}\right\}=(P\{Q>\lambda\})^{k}=(1-P\{Q<\lambda\})^{k}
$$




$$
P\left\{R_{K-k}^{\prime}\right\}=(P\{Q<\lambda\})^{K-k}
$$

where $P$ is the probability. Further, suppose $P_{0}=P\left\{H_{0}\right\}$ and $P_{1}=P\left\{H_{1}\right\}$. So, the mean reporting bits number is written as:

$$
\begin{aligned}
k_{\text {avg }} & =P_{0} \sum_{k=0}^{K-1} k\left(\begin{array}{l}
K \\
k
\end{array}\right) P\left\{\bar{R}_{K-k} \mid H_{0}\right\} \\
& +P_{1} \sum_{k=0}^{K-1} k\left(\begin{array}{l}
K \\
k
\end{array}\right) P\left\{R_{k} \mid H_{1}\right\}
\end{aligned}
$$

If $R_{0}^{\prime}$ and $R_{1}^{\prime}$ represent the probability of "No report" under hypothesis $H_{0}$ and,$H_{1}$ respectively. Then by using (14) we can write:

$$
\bar{k}=1-P_{0} R_{0}^{\prime}-P_{1} R_{1}^{\prime}
$$

where

$$
R_{0}^{\prime}=P\left\{Q<\lambda \mid H_{0}\right\}, \quad R_{1}^{\prime}=P\left\{Q<\lambda \mid H_{1}\right\}
$$

From (20) it can be written that the normalized mean reporting bits number is always $\bar{k}<1$.

\section{Decision Fusion $\boldsymbol{F}$}

Assume that the SC receiver receives $j$ group head decision where $j$ is the total groups number in the network i.e., $j=0,1, \ldots, J$, which calculated based on of $G_{j}$ decision locally reported form the cognitive users within each group. Then the last detection decision fusion, $F$, is calculated in the FC [63]:

$$
F= \begin{cases}1 & \sum_{j=1}^{J-1} f_{j} \geq 1 \\ 0 & \text { otherwise }\end{cases}
$$

$\mathrm{J}$ denoted as the active groups number in the cooperative network. These groups can be established based on beamforming in the BS or self-initiation by the nodes itself i.e., using self-organizing protocol. Let $P_{e, j}$ denote the error probability in reporting the group $j$ sensing decision and let $\gamma_{j}^{\prime}$ denote the SNR for the reporting channel in group $j . \gamma_{j}^{\prime}$ is considered as the reporting channel in the group that has the strongest SNR (with high channel quality indicator (CQI) [66]. If we use BPSK for the reporting channel, then the error probability can be written as:

$$
P_{e, j} \mid \gamma_{j}=Q\left(\sqrt{2 \gamma_{j}}\right)
$$


Therefore, the average error probability over the Rayleigh fading channel can be derived as:

$$
\begin{aligned}
P_{e, j}= & \int_{0}^{\infty} P_{e, j} \gamma_{j} f\left(\gamma_{j}\right) d \gamma_{j} \\
& =\sum_{j=0}^{J-1}\left(\begin{array}{c}
J-1 \\
j
\end{array}\right)(-1)^{J-j-1} \frac{J}{2(J-j)} \\
& \times\left(1-\sqrt{\frac{\gamma_{j}^{\prime}}{J-j+\gamma_{j}^{\prime}}}\right)
\end{aligned}
$$

where $Q($.$) is the Marcum Q- Function and J$ is the total number of cognitive group. Then from (10) and (23) the probability of detection of the network can be expressed as follows:

$$
P_{d}=\prod_{j=1}^{J} 1-P_{d, j}\left(1-P_{e, j}\right)+P_{d, j} P_{e, j}
$$

For analyzing and characterizing our cooperative sensing approach, the main performance parameter and coefficient are detection probability, $P_{d}$, and false Alarm probability, $P_{f}$, both $P_{f}$ and $P_{d}$ are performance parameters for the two design parameters distributed detection threshold and received SNR. Our proposed method assumes energy detection (ED), where the distributed detection adaptive threshold is achieved by determine the higher allowed false Alarm probability $P_{f}$. Our technique is assessed and analyzed in term of detection probability $P_{d}$ of for a certain allowed $P_{f}$.

Typically, the ultimate value for both $P_{d}$ and $P_{f}$ are 1 and 0 respectively. Though, practically the two probabilities are need be more realistic to permit a relaxation of $P_{f}$ value might be between $0.01-0.5$ and $P_{d}$ takes the range $0.1-0.99$ [55]. For protect PUs from interference by SUs, a high false Alarm probability $P_{f}$ is can be allowed than a low detection probability $P_{d}$. So, to realize that the receiveroperating characteristic (ROC) for the sensing coordinator (i.e., BS receiver) is calculated as:

$$
P_{d}=Q\left(\sqrt{2 \beta\left(\gamma_{j}\right)}, \sqrt{-2 \ln P_{f}}\right)
$$

where $Q$ is the Marcum's function and $\beta$ is a factor depend on the modulation type i.e., BPSK $\beta=1$.

\section{SIMULATION DESIGN AND ARCHITECTURE}

Distributed cooperative cognitive radio (CR) divides radios into groups, fusion node (FN) is elected from each group among all nodes which should has strongest SNR channel reporting. The 
cognitive radio nodes perform a local sensing based on ED (energy detection) and send their binary decision to the FN. Figure 5 shows the simulation modeling and DCS architecture. The fusion nodes FNS selection which was performed based on the best SNR channel reporting based on the reporting channel SNR to FC (fusion center). The flow chart in Figure 6 also shows an approach of dealing with new nodes joining or existing nodes leaving the CR network, though in our simulation, we assumed that no leaving/joining nodes with fixed architecture.

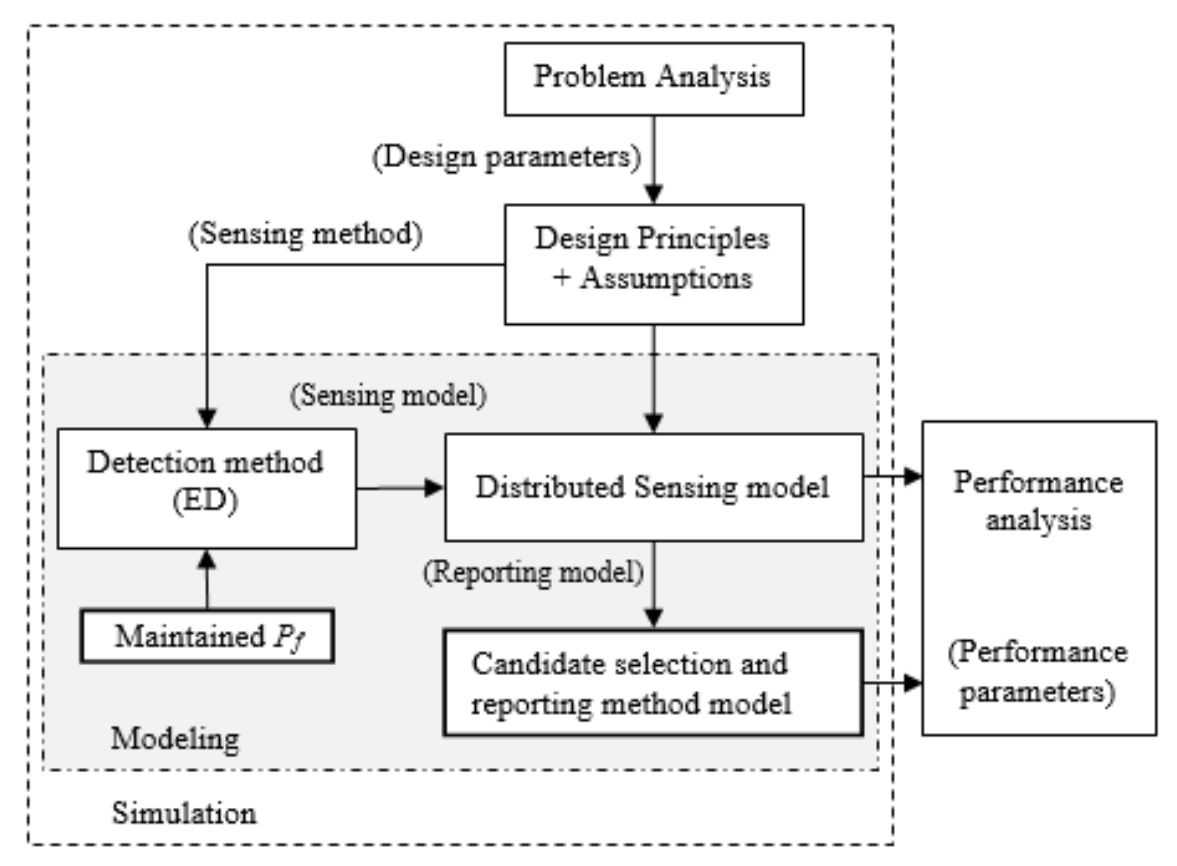

FIGURE 5: Simulation design and the DCS architecture 


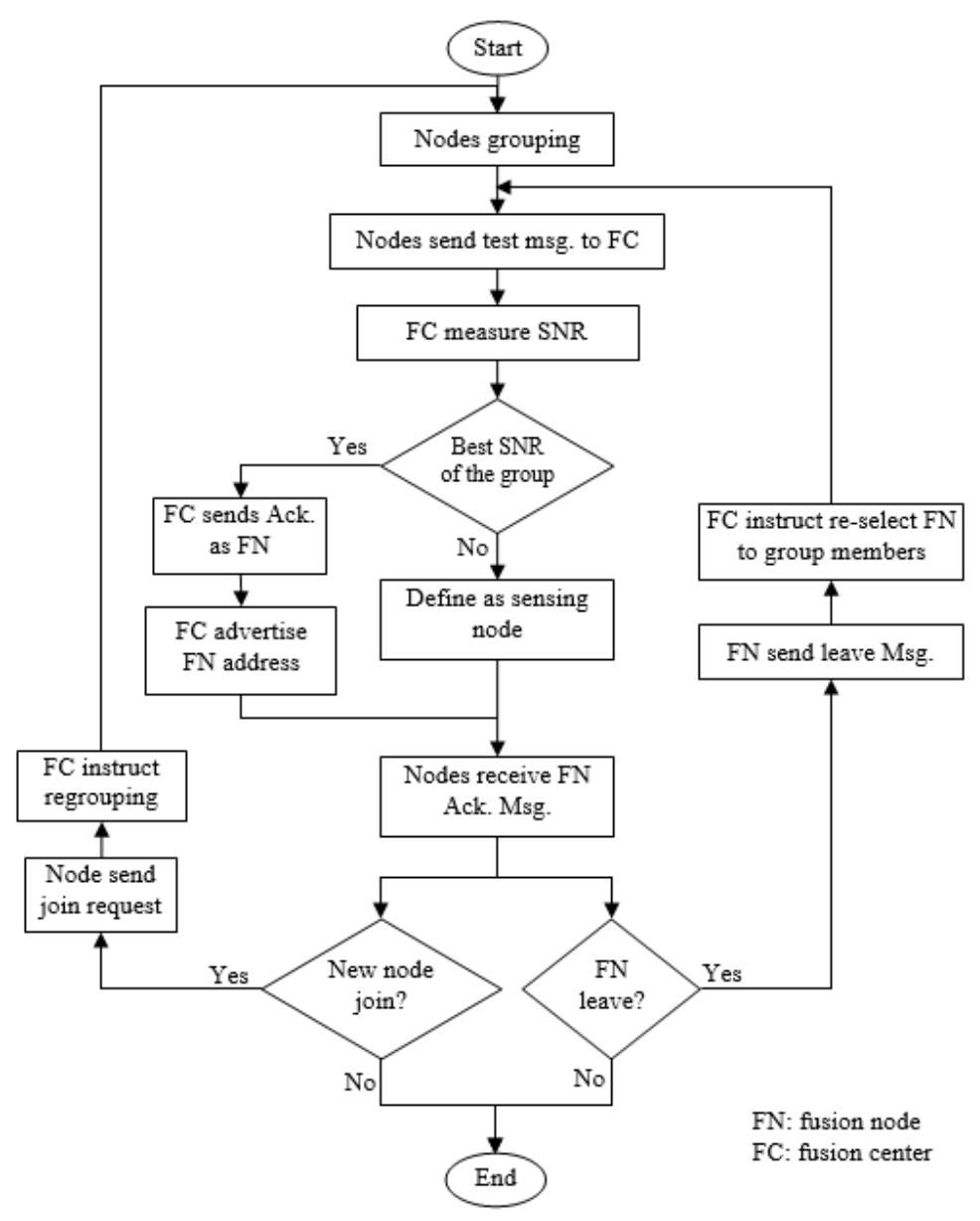

FIGURE6: Fusion node selection

The simulation architecture of the proposed method as shown in Figure 7 considers sensing groups $(J)$ with different number of nodes per group. User that has best SNR selected as group head (reporter node). Figure 8 shows 30 distributed cognitive radios with different reporting SNR (fusion node $(\mathrm{FN})$ to fusion center (FC)) where the cognitive nodes are considered an independent and identically distributed i.i.d with randomly and same probability distribution and all are mutually independent. This scatter distribution setup is used throughout the simulation. The detection thresholds are set without maintained probability of false alarm. 


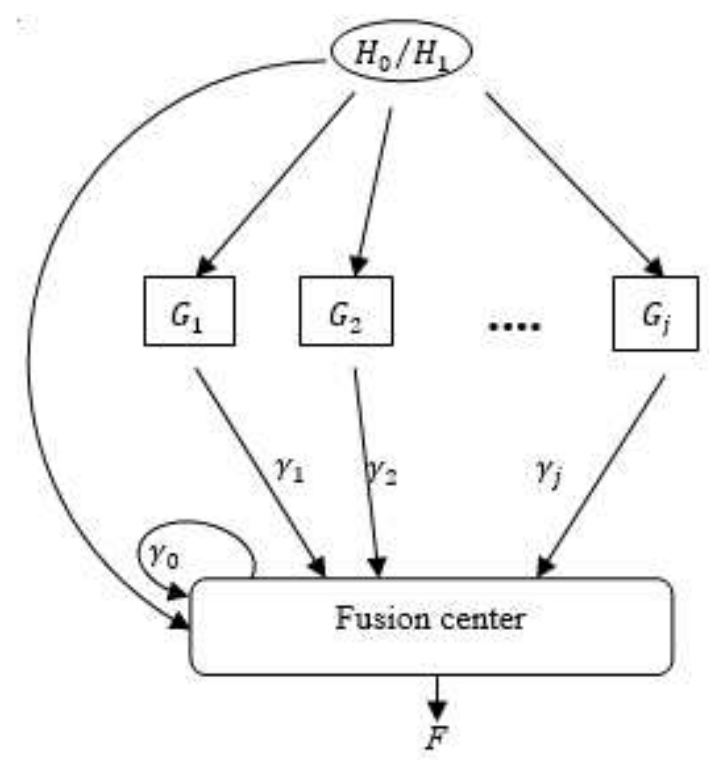

FIGURE7: Simulation Architecture

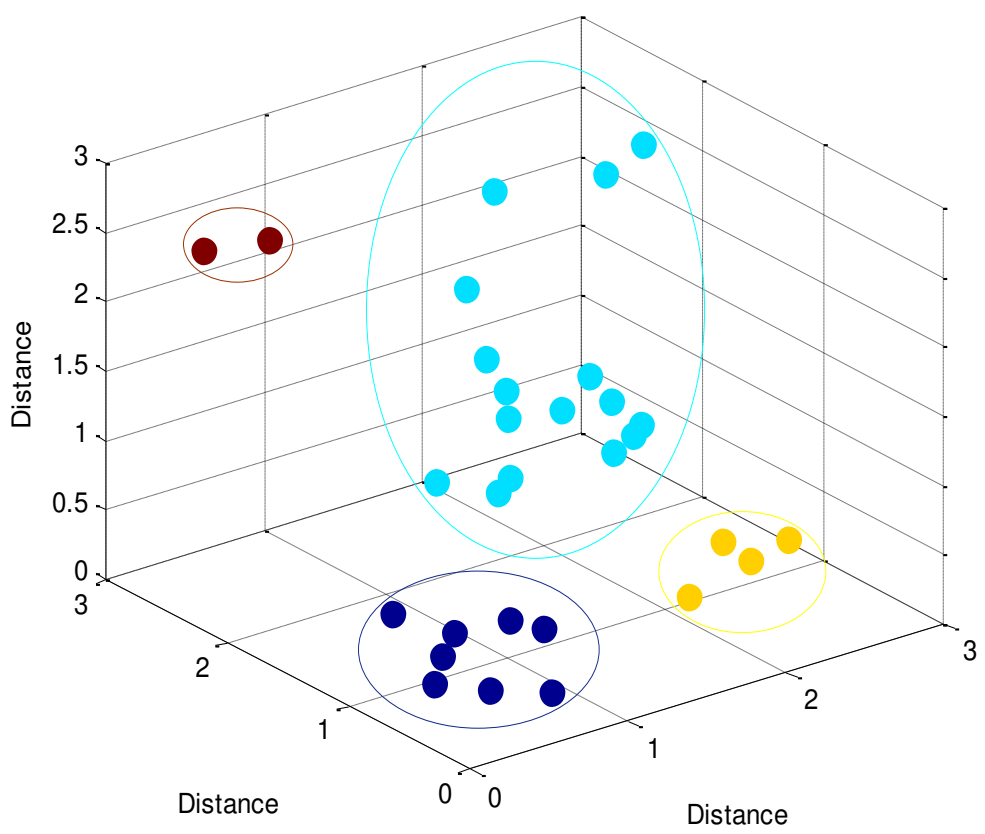

FIGURE 7: The simulation scatter distribution setup for i.i.d. 30 distributed cognitive radios with different reporting groups

\section{SIMULATION RESULT}

The Figure 8 shows the probability of detection $P_{d, j}$ vs. probability of false alarm $P_{f, j}$ for group $G_{j}$ with $K=128$ users with different SNR channels for the control / reporting to the coordinator node of cooperative sensing $(\mathrm{FN})$ from the group head $(\mathrm{GH})$. Figure 8 is also illustrating the process of 
selecting the best SNR channel for decision (less fading and shadowing affects) to improve the spectrum sensing performance. The detection probability per group $P_{d, j}$ increases to near 1 while the probability of false alarm $P_{f, j}$ is decreased. This is shown in the $\mathrm{SNR}=30 \mathrm{~dB}$, where the values $\left(P_{f, j}, P_{d, j}\right)$ were $(3.4 \times 10-7,0.97)$, which too difficult to implemented in the real situations.

Figure 9 shows the probability of error for the proposed distributed cooperative spectrum sensing (DCS) approach that derived for each group with different number of users $\{\mathrm{K}=64,128,256$ and 512$\}$. Figure 9 is also showing the error probability $\left(\mathrm{P}_{\mathrm{e}}\right)$ for fixed SNR has been enhanced and increased for the reporting phase when the cognitive users number increased within the groups. Figure 10 shows that the direct cooperative spectrum sensing can be degraded when the number of users $\mathrm{K}$ increases. Generally, the more concave the ROC curve, the better is the performance of the system i.e., high probability of detection and low probability of false match. For instance, if $\mathrm{K}=64$ with our DCS-based fusion node approach the optimal ROC values for $P_{f}$ and $P_{d}$ are 0.04 and 0.8 , respectively.

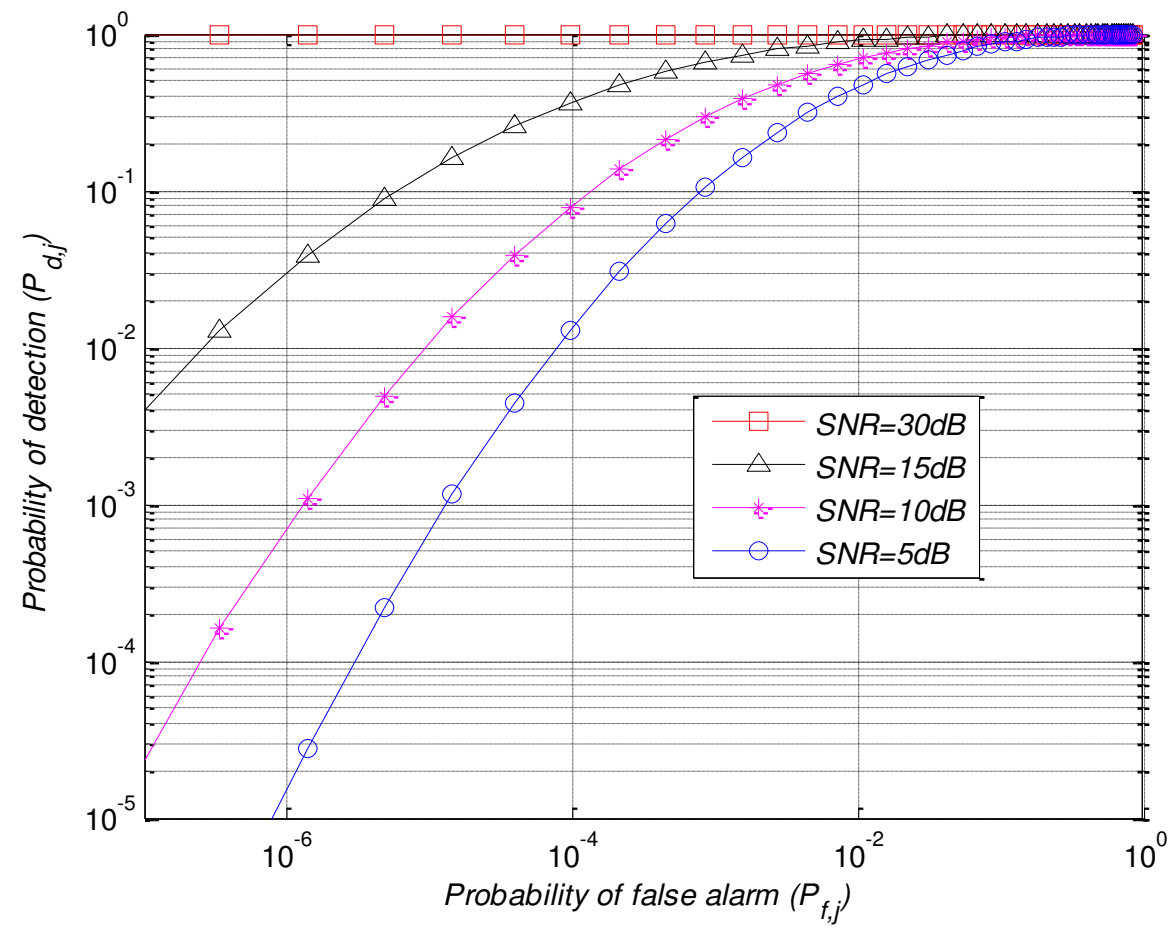

FIGURE 8. Probability of detection $P_{d, j}$ vs Probability of false alarm $P_{f, j}$ for different reporting channel SNR. 


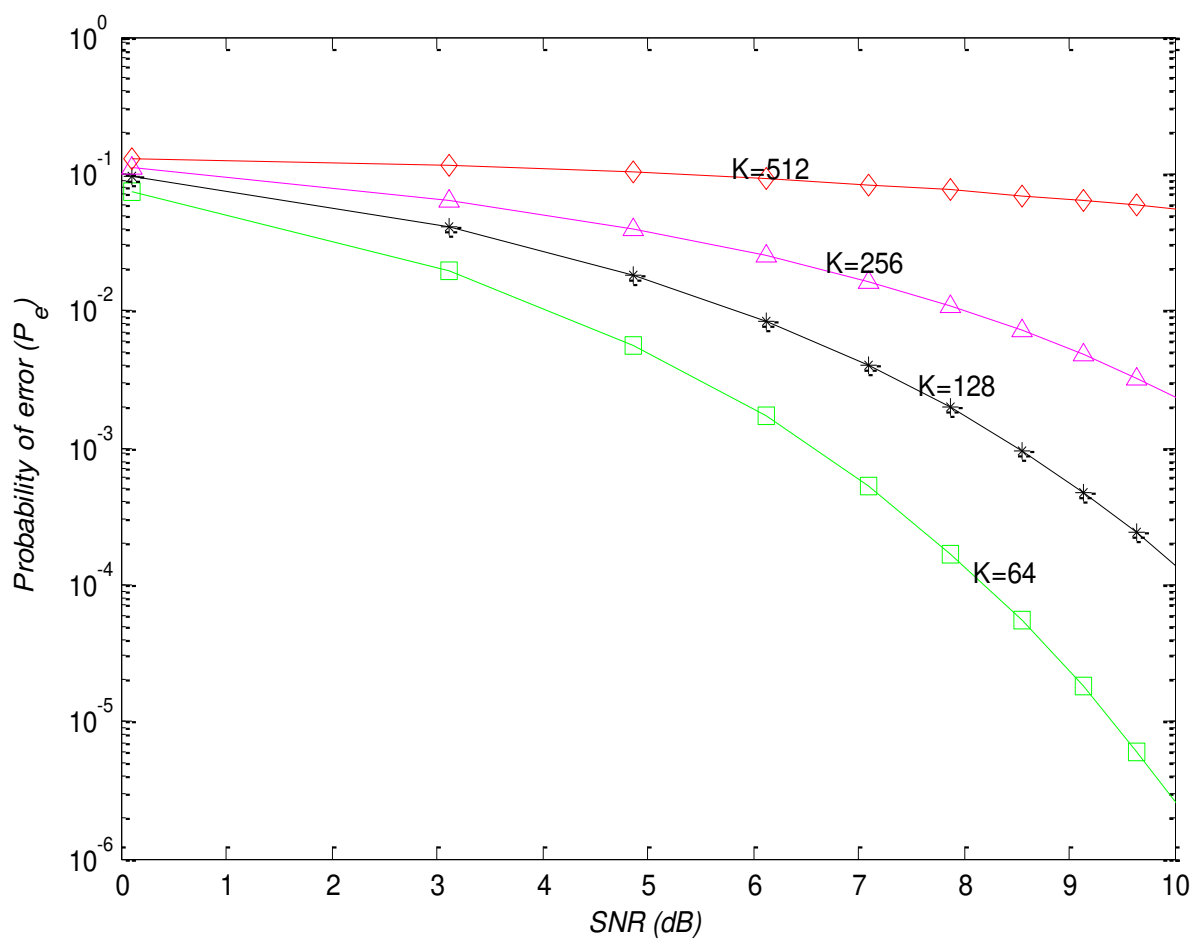

FIGURE 9. Probability of error for sensing groups allocated with different number of users $(K=64,128,156,512 ; J=4)$.

Benchmarking is conducted by using the similar number of users $\mathrm{K}$ and channel with Rayleigh fading characteristics for the previous related scheme proposed in [13] the optimal ROC values for $P_{f}$ and $P_{d}$ are 0.07 and 0.4 , respectively. Figure 11 illustrates normalization of the mean reported bits is dramatically improved when benchmarked with related works in [9], where the two quantization levels schemes show its effectiveness. The censoring phase results deterioration of false alarm probability this is due to the increases of 'No decision' section. 


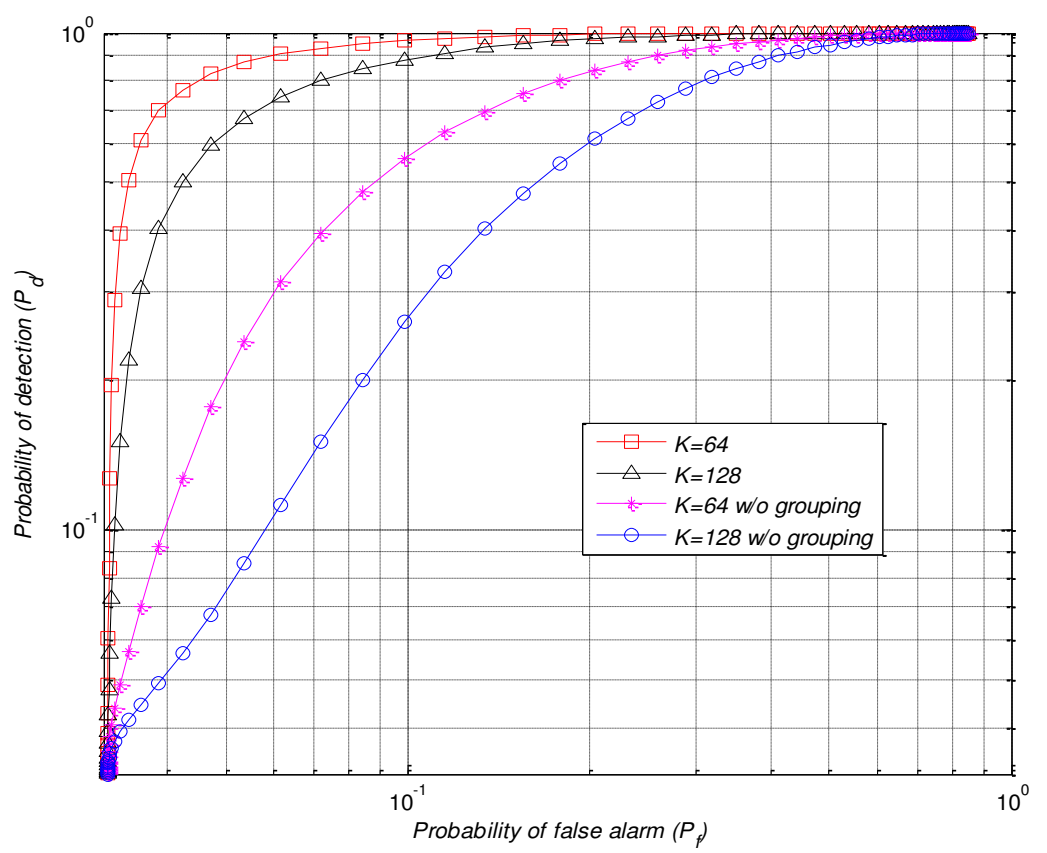

FIGURE 10. Probability of detection $P_{d}$ vs. probability of false alarm $P_{f}$ for the proposed reporting system using $S N R=10 \mathrm{~dB}$.

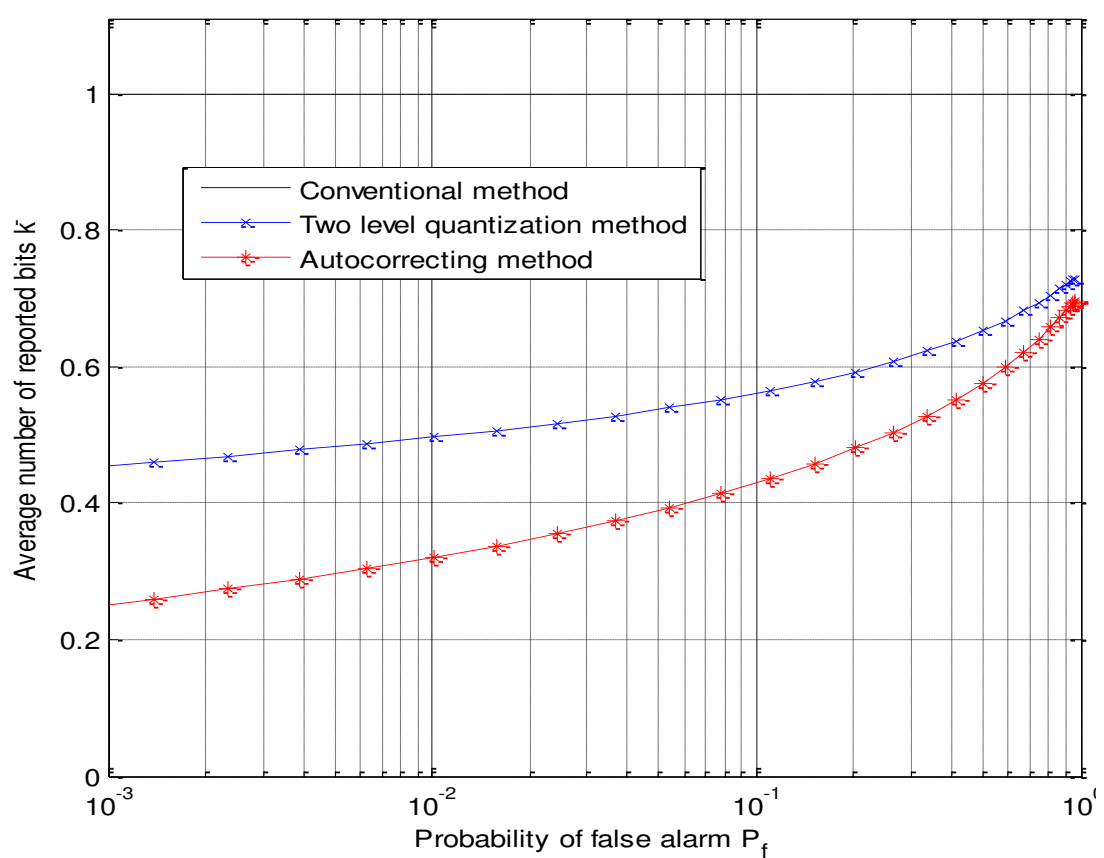

FIGURE 11. The normalized average number of sensing bits $\bar{k} \mathrm{vs} . P_{f}, N=10$, and $\mathrm{SNR}=10$ dB 
The receiver sensitivity level tells us the weakest signal that a receiver will be able to identify and process. It can be improved by reducing the noise level and bandwidth of the receiver. to have a net probability of false alarm $P_{f}$ to be around 0.1 , the threshold should be set three standard deviations away from the mean.

This places the threshold at: $\alpha_{\max }^{\lambda 1}$ where $\lambda 1=2_{w}^{2}\left(1+\sqrt{2\left(9+\ln \left(G_{j}\right) / N\right.}\right)$. The factor $\alpha_{\max }^{2_{w}^{2}}$ is the worst-case noise variance power. If we bound system performance by assuming that all provided samples to Cognitive radio (CR) using the best SNR channel. In that case the false alarm probability threshold can be set at: $\alpha_{\text {max }}^{\lambda 2}$, where $\lambda 2=2_{w}^{2}\left(1+3 \sqrt{2 / G_{j} N}\right)$ and $G_{j}$ is the CR nodes number in the group.

To perceive the variation in sensitivity; we simulated two sensing groups with the same reporting channel with SNR, $\gamma^{\prime}=10 \mathrm{~dB}$. Number of users in all groups was varied and the effect of $90 \%$ and 95\% radio sensitivity for a detection probability was examined. The effect of cooperation process on the sensitivity threshold $(m)$ of single radio can be shown in Figure 12 illustrates un-bounded enhancement of sensitivity threshold in $m$ watt with various number of users Sensitivity variation with number of users and two different detection probability $\left(P_{f}=0.1\right)$.

Our ultimate aim is the sensing time minimization on cooperative environment. To calculate the detection probability, average power for primary channel has to be inspected. In addition to that energy detection (ED) in the channel, which is vital coefficient for detection performance. Hereafter it minimizes the sensing phase duration for efficient utilize for radio resources.

In addition to that, threshold value and SNR (signal-to-noise ratio) value has to be determined for identifying the probability of detection in a renewal process. Hence the probability of detection would be efficient based on two major factors with less interference and primary channel transition state. These are two main factors for efficient probability of detection in vehicular networks. In addition to that, the fake SU issue is also a major concern. However, in cooperative spectrum sensing involvement, fake user in graduation is decreased due to a centralized approach. 


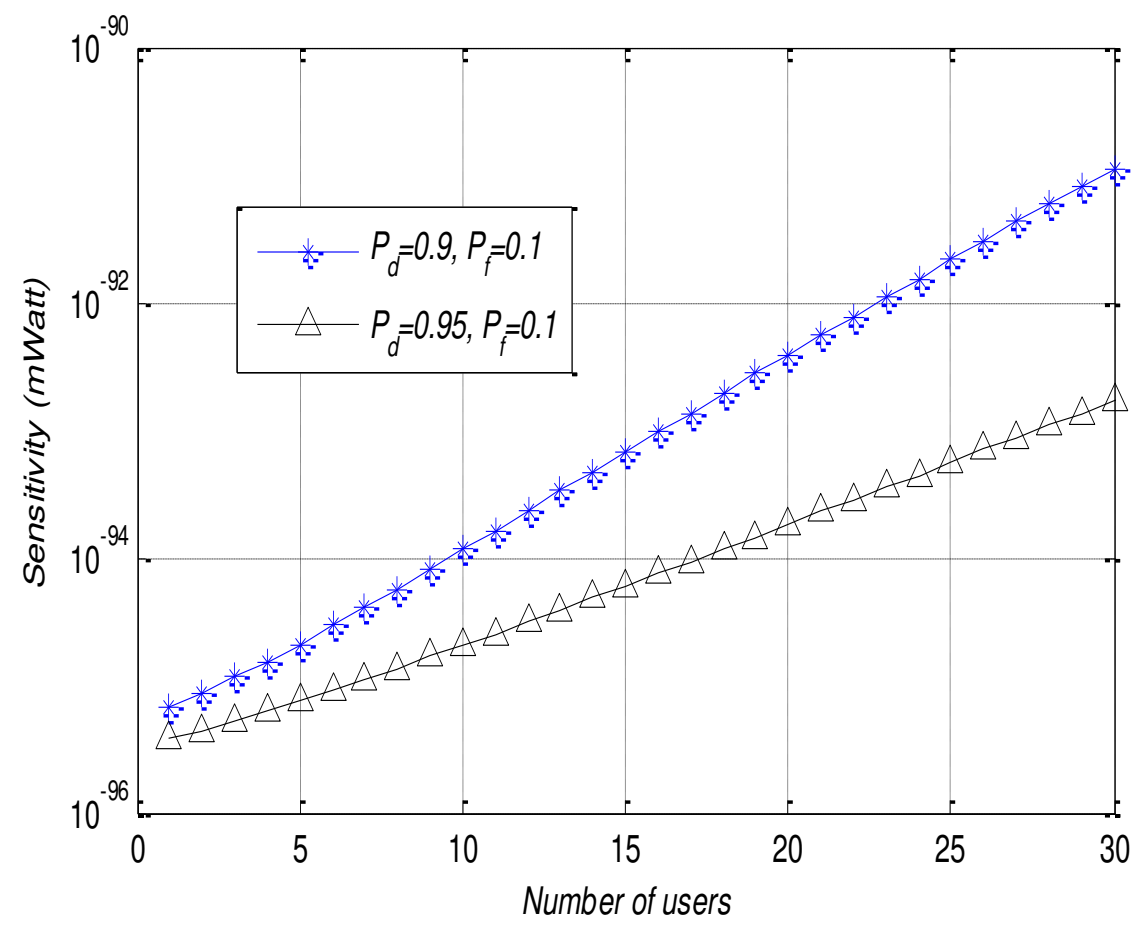

FIGURE 12. Un-bounded enhancement of sensitivity threshold in $\boldsymbol{m}$ watt with various number of users and two different detection probability $\left(P_{f}=0.1\right)$.

\section{B. Fusion Analysis}

Fusion decision is joining of CR sensing data or data processed from different CR nodes, where data usually has lower uncertainty when it used individual node. The term uncertainty enhancement means more complete, dependable, or accurate. Figure 13 illustrates the analysis and compare between OR-rule (one-out-of-N) and AND-rule for different number of candidates' nodes per fusion decision group. OR-rule is resultant of emerging of all mutually exclusive sensing reports in a group of cooperative distributed spectrum detection process compared to AND-rule. The results show that when the false alarm probability is less than 0.04, AND-rule decision based is out-perform OR-rule based decision. 


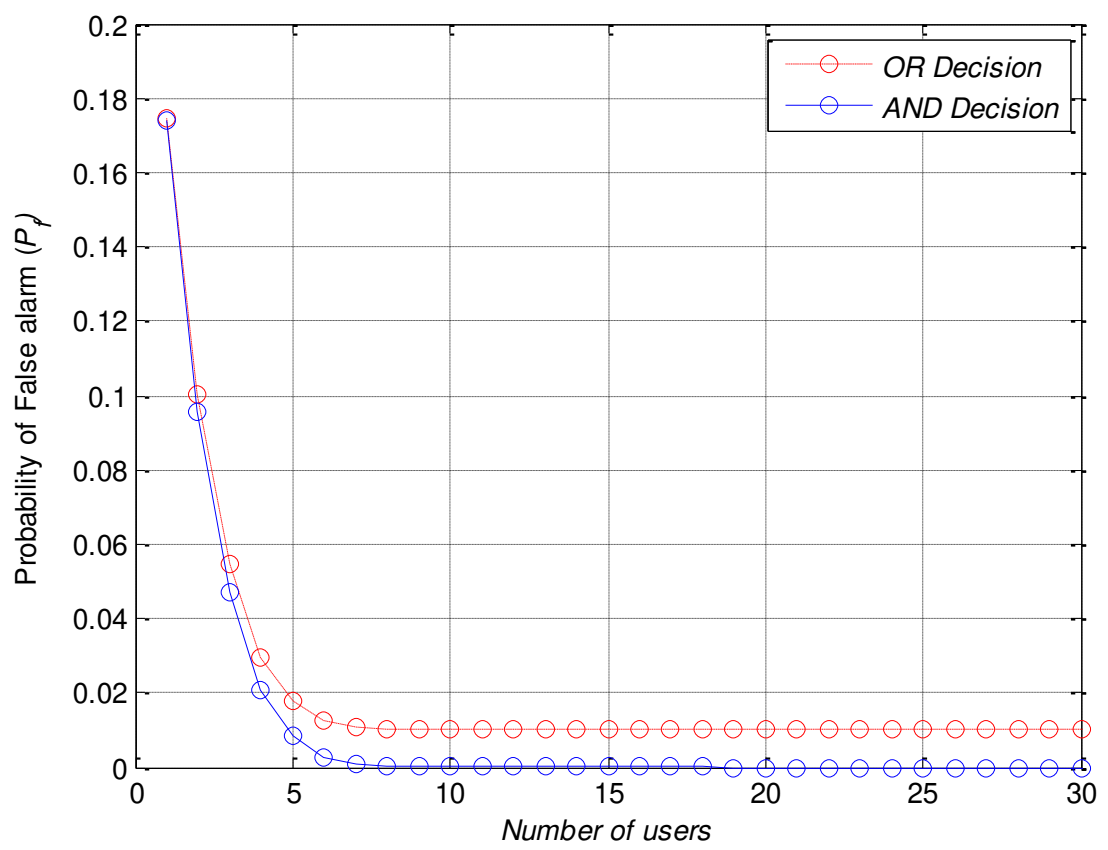

\section{FIGURE 13. Drawback of the distributed cooperative spectrum sensing based OR-rule compared to AND-rule.}

\section{CONCLUSIONS}

The performance of the cooperative spectrum sensing and reporting in cognitive radio network can be improved and overcome fading impact by using a distributed group sensing approach. This is by exploiting the reporting channel selection diversity to enhance reporting stage. In so far as the CR network nodes growing, the proposed cooperative scheme would have shortened the protocol messages overhead. The paper also proposed an auto correction scheme for reported bits error. The normalized average number of reported bits is also greatly reduced and enhanced with an efficiently. The simulation results examined and analyzed the proposed algorithm performance in cooperative spectrum sensing with distributed fusion selection, the normalized reported bits average number has been verified by simulation result which demonstrates notable reduction in reporting bits compared with the existing non-cooperative method without reduction in performance and network throughput. The numerical simulation and analytical derivation recommend that the algorithm significantly enhance the detection probability and the number of nodes that cooperate in fusion decision is reduced also. The combination of distributed fusion scheme with the conventional ED (energy detection) was modeled to accomplish best reporting and sensing probabilities. through simulation model, by varying the sensing time in nodes with hierarchical multilevel reporting procedure, the network throughput is optimized using receiver operating characteristic curve. For further research works other parameters such as clustering and grouping procedure, users per group optimization, space diversity, and coverage area can be considered and studied. 


\section{Declarations}

Funding: This research was supported by Taif University Researchers Supporting Project Number (TURSP-2020/216), Taif University, Taif, Saudi Arabia

Conflicts of interest/Competing interests: All authors certify that they have no affiliations with or involvement in any organization or entity with any financial interest or non-financial interest in the subject matter or materials discussed in this manuscript.

Availability of data and material: The datasets generated during and/or analysed during the current study are available from the corresponding author on reasonable request.

Code availability: The code generated during the current study are available from the corresponding author on reasonable request.

\section{Authors contribution}

- Conceptualization, Rania A. Mokhtar, Rashid A. Saeed

- Data curation, Hesham Alhumyani, Rashid A. Saeed

- Formal analysis, Rania A Mokhtar, Hesham Alhumyani

- Funding acquisition, Rashid A. Saeed, Hesham Alhumyani

- The investigation, All Authors

- Methodology, Rania A. Mokhtar, Rashid A. Saeed

- Project administration, All Authors

- Resources, Rania A. Mokhtar, Rashid A. Saeed

- Software, All Authors

- Supervision, Rashid A Saeed,Hesham Alhumyani

- Validation, Hesham Alhumyani, Rashid A. Saeed

- Visualization, Rania A. Mokhtar, Rashid A. Saeed

- Writing - original draft, All authors

- Writing - review editing - All authors 


\section{REFERENCES}

[1] M. Karimi, S. Mohammad, S. Sadough, M. Torabi, “Optimal Cognitive Radio Spectrum Access with Joint Spectrum Sensing and Power Allocation", IEEE Wireless Communications Letters, Volume: 9, Issue: 1, 2020.

[2] Peter J. Smith, Rajitha Senanayake, Pawel A. Dmochowski, Jamie S. Evans, "Distributed Spectrum Sensing for Cognitive Radio Networks Based on the Sphericity Test”, IEEE Transactions on Communications, Volume: 67, Issue: 3, 2019.

[3] Min Deng, Bin-Jie Hu, "LLR Based Spatiotemporal Cooperative Spectrum Sensing Schemes for Cognitive Radios", IEEE Inter. Conf. on Sig. Proc., Comm. and Comp. (ICSPCC2015), 2015.

[4] J. Bodart, S. Gishkori, J. Verlant-Chenet, L. Lampe, F. Horlin, "Multiband Spectrum Sensing for Cognitive Radios Based on Distributed Compressed Measurements", IEEE International Conference on Communications (ICC), 2015.

[5] Čabrić1 D., Mubaraq S., Willkomm D., Brodersen1 R., Wolisz A., “A Cognitive Radio Approach for Usage of Virtual Unlicensed Spectrum," Inter. Workshop on UWB Sys. Joint with Conf. on UWB Sys. and Tech. (UWBST \& IWUWBS 2005), pp. 50-54, 2005.

[6] F. F. Digham, M.-S.Alouini, and M. K. Simon, "On The Energy Detection Of Unknown Signals Over Fading Channels," in Proc. IEEE ICC, Anchorage, AK, USA, pp. 35753579, May 2003.

[7] Xin Liu, Min Jia, Zhenyu Na, Weidang Lu, Feng Li, "Multi-Modal Cooperative Spectrum Sensing Based on Dempster-Shafer Fusion in 5G-Based Cognitive Radio", IEEE Access, Volume: 6, 2018.

[8] W. Zhang, and Letaief K. B., "Cooperative Spectrum Sensing for Cognitive Radios Under Bandwidth Constraints," IEEE Intern. Wireless Comm. and Netw. Conf. (WCNC2007), pp. 1-5, 2007.

[9] Wenshan Yin, Hao Chen, "Decision-Driven Time-Adaptive Spectrum Sensing in Cognitive Radio Networks", IEEE Transactions on Wireless Communications, Volume: 19, Issue: 4, 2020.

[10] Seleman Daudi Arthur Nkalango, Hui Zhao, Yuxin Song, Tianqi Zhang, "Energy Efficiency Under Double Deck Relay Assistance on Cluster Cooperative Spectrum Sensing in Hybrid Spectrum Sharing”, IEEE Access, Volume: 8, 2020. 
[11] Brijesh Soni, Dhaval K. Patel, Miguel López-Benítez, "Long Short-Term Memory Based Spectrum Sensing Scheme for Cognitive Radio Using Primary Activity Statistics", IEEE Access, Volume: 8, 2020.

[12] Junhee Kim, Jihwan P. Choi, "Sensing Coverage-Based Cooperative Spectrum Detection in Cognitive Radio Networks", IEEE Sensors Journal, Volume: 19, Issue: 13, Year: 2019.

[13] Rashid A. Saeed, "Spectrum Sensing and Sharing for Cognitive Radio" published by River Publishers of Denmark in the book with title "Planning and Optimization of $3 G$ and 4G Wireless Network” ISBN: 978-87-92329-24-0, page 219-259, January 2009.

[14] Juan Augusto Maya, Leonardo Rey Vega, Cecilia G. Galarza, “A Locally Optimal Soft Linear-Quadratic Scheme for CR Systems in Shadowing Environments", IEEE Wireless Communications Letters, Volume: 5, Issue: 3, 2016.

[15] Faroq Awin, Esam Abdel-Raheem, Kemal Tepe, "Blind Spectrum Sensing Approaches for Interweaved Cognitive Radio System: A Tutorial and Short Course", IEEE Communications Surveys \& Tutorials, Volume: 21, Issue: 1, 2019.

[16] Ahmed Badawy, Ahmed El Shafie, Tamer Khattab, "On the Performance of Quickest Detection Spectrum Sensing: The Case of Cumulative Sum", IEEE Communications Letters, Volume: 24, Issue: 4, Year: 2020.

[17] M. A. Al-Jarrah, A. Al-Dweik, S. S. Ikki, E. Alsusa, "Spectrum-Occupancy Aware Cooperative Spectrum Sensing Using Adaptive Detection", IEEE Sys. Jour., Volume: 14, Issue: $2,2020$.

[18] Haifeng Lin, Lin Du, Yunfei Liu, "Soft Decision Cooperative Spectrum Sensing with Entropy Weight Method for Cognitive Radio Sensor Networks ”, IEEE Access, Volume: 8, 2020.

[19] F. Jalali, A. Zaimbashi, “Cognitive Radio Spectrum Sensing Under Joint TX/RX I/Q Imbalance and Uncalibrated Receiver”, IEEE Sys. Jour., Volume: 14, Issue: 1, 2020.

[20] Grigorios Kakkavas, Konstantinos Tsitseklis, Vasileios Karyotis, Symeon Papavassiliou, “A Software Defined Radio Cross-Layer Resource Allocation Approach for Cognitive Radio Networks: From Theory to Practice", IEEE Transactions on Cognitive Communications and Networking, Volume: 6, Issue: 2, Year: 2020.

[21] Rashid A. Saeed, A. F. Ismail, Mohammad Kamrul Hasan, Rania Mokhtar, Sajda K. A. Salih, Wahida Hashim, "Throughput Enhancement for WLAN TV White Space in Coexistence of IEEE 802.22", Indian Journal of Science and Technology (IJSC), Volume 8, Issue 11, DOI: 10.17485/ijst/2015/v8i11/71783, June 2015 
[22] R. Mokhtar, Rashid A. Saeed, R Alsaqour, Y Abdallah, "Study on Energy Detectionbased Cooperative Sensing in Cognitive Radio Networks", Journal of Networks (JNW), Vol. 8, No. 6, Pp. 1255-1261, June 2013, doi:10.4304/jnw.8.6.1255-1261

[23] Akinbode A. Olawole, Fambirai Takawira, Olutayo O. Oyerinde, "Cooperative Spectrum Sensing in Multichannel Cognitive Radio Networks with Energy Harvesting", IEEE Access, Volume: 7, 2019.

[24] Rashid A. Saeed, “TV White Space Spectrum Technologies: Regulations, Standards, and Applications", CRC Press, USA, ISBN: 9781439848791, Sept. 2011

[25] Peter J. Smith, Rajitha Senanayake, Pawel A. Dmochowski, Jamie S. Evans, "Distributed Spectrum Sensing for Cognitive Radio Networks Based on the Sphericity Test”, IEEE Transactions on Communications, Volume: 67, Issue: 3, 2019.

[26] Rashid A. Saeed, Tuncer Baykas, Mark Cummings, Envia, Hyunduk Kang, Mika Kasslin, Joe Kwak, Richard Paine, Alex Reznik and Stephen J. Shellhammer, "A Standard for TV White Space Coexistence: Technical Challenges and Solution Approaches”, IEEE Wireless Communication Magazine, Vol. 19 Issue: 2, pp 10 - 22 Feb. 2012.

[27] Xuanheng Li, Haichuan Ding, Yuguang Fang, “A Data-Driven Cost-Effective Session-Oriented Cognitive Radio Transmission Scheme Under Spectrum Uncertainty”, IEEE Transactions on Vehicular Technology, Volume: 68, Issue: 12, 2019.

[28] Zhi Zhang, Xianbin Wen, Haixia Xu, Liming Yuan, "Sensing Nodes Selective Fusion Scheme of Spectrum Sensing in Spectrum-Heterogeneous Cognitive Wireless Sensor Networks", IEEE Sensors Journal, Volume: 18, Issue: 1, 2018.

[29] Peixiang Cai, Yu Zhang, "Intelligent Cognitive Spectrum Collaboration: Convergence of Spectrum Sensing, Spectrum Access, and Coding Technology", Intelligent and Converged Networks, Volume: 1, Issue: 1, 2020.

[30] Qi Yang, Yi-Feng Huang, Yin-Chen Yen, Ling-Yu Chen, Hsiao-Hwa Chen, XueMin Hong, Jiang-Hong Shi, Liangmin Wang, "Location Based Joint Spectrum Sensing and Radio Resource Allocation in Cognitive Radio Enabled LTE-U Systems”, IEEE Transactions on Vehicular Technology, Volume: 69, Issue: 3, 2020.

[31] Lin Zhang, Ying-Chang Liang, Joint Spectrum Sensing and Packet Error Rate Optimization in Cognitive IoT, IEEE Internet of Things Journal, Volume: 6, Issue: 5, 2019. 
[32] Shuai Yuan, Lei Li, Chunxiao Chigan, "On MMD-Based Secure Fusion Strategy for Robust Cooperative Spectrum Sensing", IEEE Transactions on Cognitive Communications and Networking, Volume: 5, Issue: 3, 2019.

[33] Hongning Li, Yu Gu, Jiexiong Chen, Qingqi Pei, "Speed Adjustment Attack on Cooperative Sensing in Cognitive Vehicular Networks", IEEE Access, Volume: 7, 2019.

[34] J. Lee, H. Baek, J. Lim, "Cooperative Sensing Scheme for Acquisition of Rotational Synchronization of Radar”, IEEE Sys. Jour., Volume: 14, Issue: 2, 2020.

[35] Iickho Song, Dongjin Kim, Seungwon Lee, Seokho Yoon, "Selection-Based Detectors and Fusion centers (FCs) For Cooperative Cognitive Radio Networks in Heavy-Tailed Noise Environment", Journal of Communications and Networks, Volume: 19, Issue: 3, 2017.

[36] Sara A. Attalla, Karim G. Seddik, Amr A. El-Sherif, Sherif I. Rabia, "Hybrid ARQCQI Feedback-Based Access Scheme in Cognitive Radio Networks", IEEE Transactions on Cognitive Communications and Networking, Volume: 6, Issue: 2, 2020.

[37] Charu Bhatnagar, Anjali Potnis, Prshant Dwivedy, Sunil Kumar Meena, "Performance Analysis and Optimization Schemes for Cooperative Spectrum Sensing and Information Fusion for Cognitive Radio: A Survey, 2017 1st International Conference on Electronics, Materials Engineering and Nano-Technology (IEMENTech), 2017.

[38] Jingwen Tong, Ming Jin, Qinghua Guo, Youming Li, "Cooperative Spectrum Sensing: A Blind and Soft Fusion Detector", IEEE Transactions on Wireless Communications, Volume: 17, Issue: 4, 2018.

[39] Hamid Eltom, Sithamparanathan Kandeepan, Ying-Chang Liang, Robin J. Evans, "Cooperative Soft Fusion for HMM-Based Spectrum Occupancy Prediction”, IEEE Communications Letters, Volume: 22, Issue: 10, 2018.

[40] Shunchao Zhang, Yonghua Wang, Pin Wan, Jiawei Zhuang, Yongwei Zhang, Yi Li, "Clustering Algorithm-Based Data Fusion Scheme for Robust Cooperative Spectrum Sensing”, IEEE Access, Volume: 8, 2020.

[41] Marwan Hadri Azmi, Harry Leib, "Multichannel Cooperative Spectrum Sensing That Integrates Channel Decoding with Fusion-Based Decision", IEEE Transactions on Aerospace and Electronic Systems, Volume: 54, Issue: 4, 2018.

[42] Srinivas Nallagonda, Godugu Kiran Kumar, Ashok Kumar Nallagonda, "Comprehensive Performance Analysis of Data-Fusion Aided Cooperative Cognitive 
Radio Network Over H - M Fading Channel”, IET Communications, Volume: 13, Issue: $16,2019$.

[43] Akinbode A. Olawole, Fambirai Takawira, Olutayo O. Oyerinde, "Fusion Rule and Cluster Head Selection Scheme In Cooperative Spectrum Sensing", IET Communications, Volume: 13, Issue: 6, 2019.

[44] Rashid Mustafa, Reena Rathee Jaglan, Sunil Agrawal, "Decision-fusion-based reliable CSS scheme in CR networks", IET Communications, Volume: 13, Issue: 7, 2019.

[45] Keyu Wu, Min Tang, Chintha Tellambura, Dongtang Ma, "Cooperative Spectrum Sensing as Image Segmentation: A New Data Fusion Scheme”, IEEE Communications Magazine, Volume: 56, Issue: 4, 2018.

[46] Pankaj Verma, "Weighted Fusion Scheme for Cooperative Spectrum Sensing, International Conference on Industry 4.0 Technology (I4Tech), 2020.

[47] Mostafa E. Youssef, Shahzad Nasim, Sarwar wasi, Ulfat Khisal, Arsalan Khan, "Efficient Cooperative Spectrum Detection in Cognitive Radio Systems Using Wavelet Fusion”, IEEE Inter. Conf. on Comp., Elect. and Elect. Eng. (ICE_Cube2018), 2018.

[48] Girraj Sharma, Ritu Sharma, "Performance Comparison Of Hard And Soft Fusion Techniques For Energy Efficient CSS In Cognitive Radio”, IEEE Inter Conf. on Adv. Comp. and Telecomm. (ICACAT2018), 2018.

[49] Ashwini Kumar Varma, Debjani Mitra, “A Neural Network Approach to Decision Fusion for Wideband Cooperative Sensing, 2018 Conference on Information and Communication Technology (CICT), 2018.

[50] Gaurav Verma, Vinayak Dhage, Sudakar Singh Chauhan, "Analysis Of Combined Data-Decision Fusion Scheme For Cognitive Radio Networks", 2018 2nd International Conference on Inventive Systems and Control (ICISC), 2018.

[51] Ayman A. El-Saleh, Mahmoud A. M. Albreem, Tauseef Rasheq Ahad, Waziha Raquib, "Cross entropy algorithm for improved soft fusion-based cooperative spectrum sensing in cognitive radio networks", IEEE MENA Comm. Conf. (MENACOMM2018), 2018.

[52] Arpita Chakraborty, Jyoti Sekhar Banerjee, Abir Chattopadhyay, "Non-Uniform Quantized Data Fusion Rule Alleviating Control Channel Overhead for Cooperative Spectrum Sensing in Cognitive Radio Networks”, 2017 IEEE 7th International Advance Computing Conference (IACC), 2017. 
[53] Zafar Iqbal, Saeid Nooshabadi, Khaled Jadi, Ahmad Ghasemi, "Sensor Cooperation and Decision Fusion to Improve Detection in Cognitive Radio Spectrum Sensing”, $9^{\text {th }}$ IEEE Annual Ubiq. Comp., Elect. \& Mobile Comm. Conf. (UEMCON2018), 2018.

[54] Catherine Paola Méndez Rangel, Luiz Alencar Reis da Silva Mello, “Analysis Of Performance of Fusion Rules For Cooperative Spectrum Sensing”, IEEE L-A Conf. on Comm. (LATINCOM2019), 2019.

[55] V. Balaji, "Reinforcement Learning Based Decision Fusion Scheme for Cooperative Spectrum Sensing in Cognitive Radios", International Conference on Wireless Communications, Signal Processing and Networking (WiSPNET), 2018.

[56] Junhai Luo, Xiaoting He, Man Wu, Yanping Chen, Yang Yang, “An Optimal Bit Allocation Scheme for Cooperative Spectrum Sensing in Cognitive Radio Networks", 22th International Conference on Information Fusion (FUSION), 2019.

[57] Suresh Kumar Balam, P. Siddaiah, Srinivas Nallagonda, "Throughput Analysis of Cooperative Spectrum Sensing with Hard-decision Fusion over Generalized $\kappa-\mu$ Fading Channel" IEEE 2nd Inter. Conf. on Adv. in Elec., Comp. and Comm. (ICAECC2018), 2018.

[58] Oluyomi Simpson, Yichuang Sun, "A Stochastic based Physical Layer Security in Cognitive Radio Networks: Cognitive Relay to Fusion Center", IEEE 38th Int. Performance Comp. and Comm. Conf. (IPCCC 2019), 2019.

[59] Raefga Elgadi, Allaa R. Hilal, Otman Basir, "Intelligent Hybrid Cooperative Spectrum Sensing: A Multi-Stage Decision Fusion Approach", IEEE International Conference on Systems, Man, and Cybernetics (SMC), 2017.

[60] Rausley A. A. de Souza, Lucas dos Santos Costa, Eduardo Moreira de Almeida, "A Novel Decision Fusion Periodogram-based Algorithm for Centralized Cooperative Spectrum Sensing Under Errors at the Report Channel”, 13th European Conference on Antennas and Propagation (EuCAP), 2019.

[61] Shuai Yuan, Lei Li, Chunxiao Chigan, "Maximum Mean Discrepancy Based Secure Fusion Strategy for Robust Cooperative Spectrum Sensing", IEEE International Conference on Communications (ICC), 2018.

[62] Srinivas Nallagonda, Y. Rakesh Kumar, Pasham Shilpa, “Analysis of Hard-Decision and Soft-Data Fusion Schemes for Cooperative Spectrum Sensing in Rayleigh Fading Channel”, 2017 IEEE 7th International Advance Computing Conference (IACC), 2017.

[63] Hang Liu, Xu Zhu, Takeo Fujii, "Ensemble Deep Learning Based Cooperative Spectrum Sensing with Semi-soft Stacking Fusion Center”, 2019 IEEE Wireless Communications and Networking Conference (WCNC), 2019. 
[64] Hang Liu, Xu Zhu, Takeo Fujii, "Ensemble Deep Learning Based Cooperative Spectrum Sensing with Stacking Fusion Center”, 2018 Asia-Pacific Signal and Information Processing Association Annual Summit and Conference (APSIPA ASC), 2018.

[65] Meenakshi Awasthi, Madhav J. Nigam, Vijay Kumar, Energy Efficient Hard Decision Fusion Rules for Fading and Non-Fading Environment”, 2017 IEEE Region 10 Conference (TENCON), 2017. 
Figures

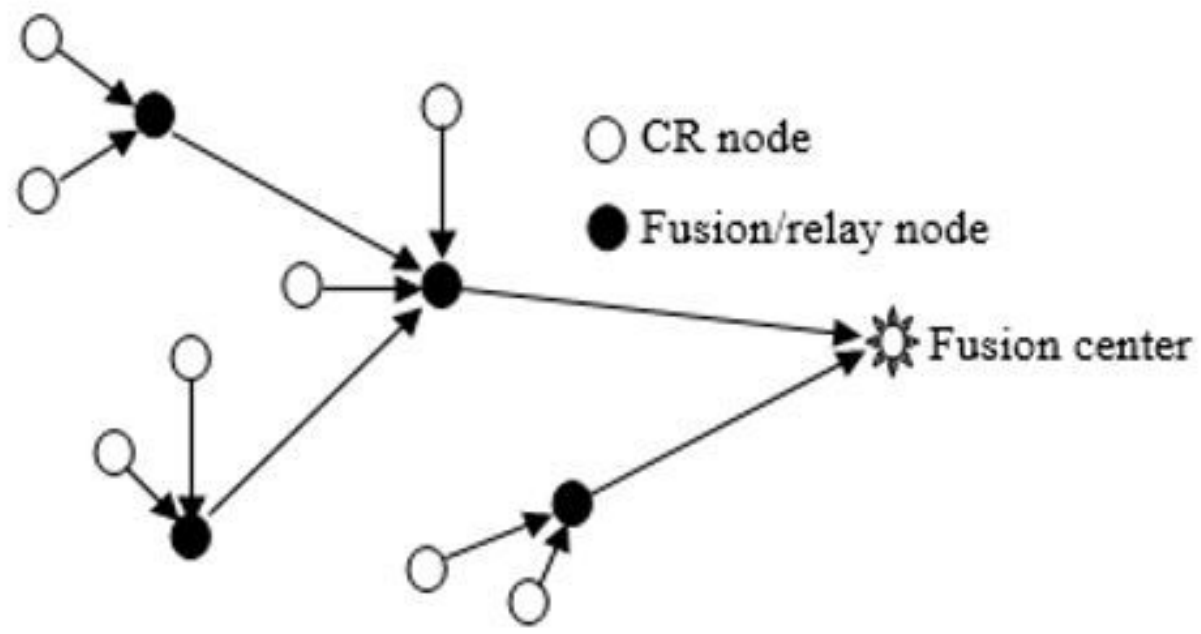

Figure 1

Network Architecture 


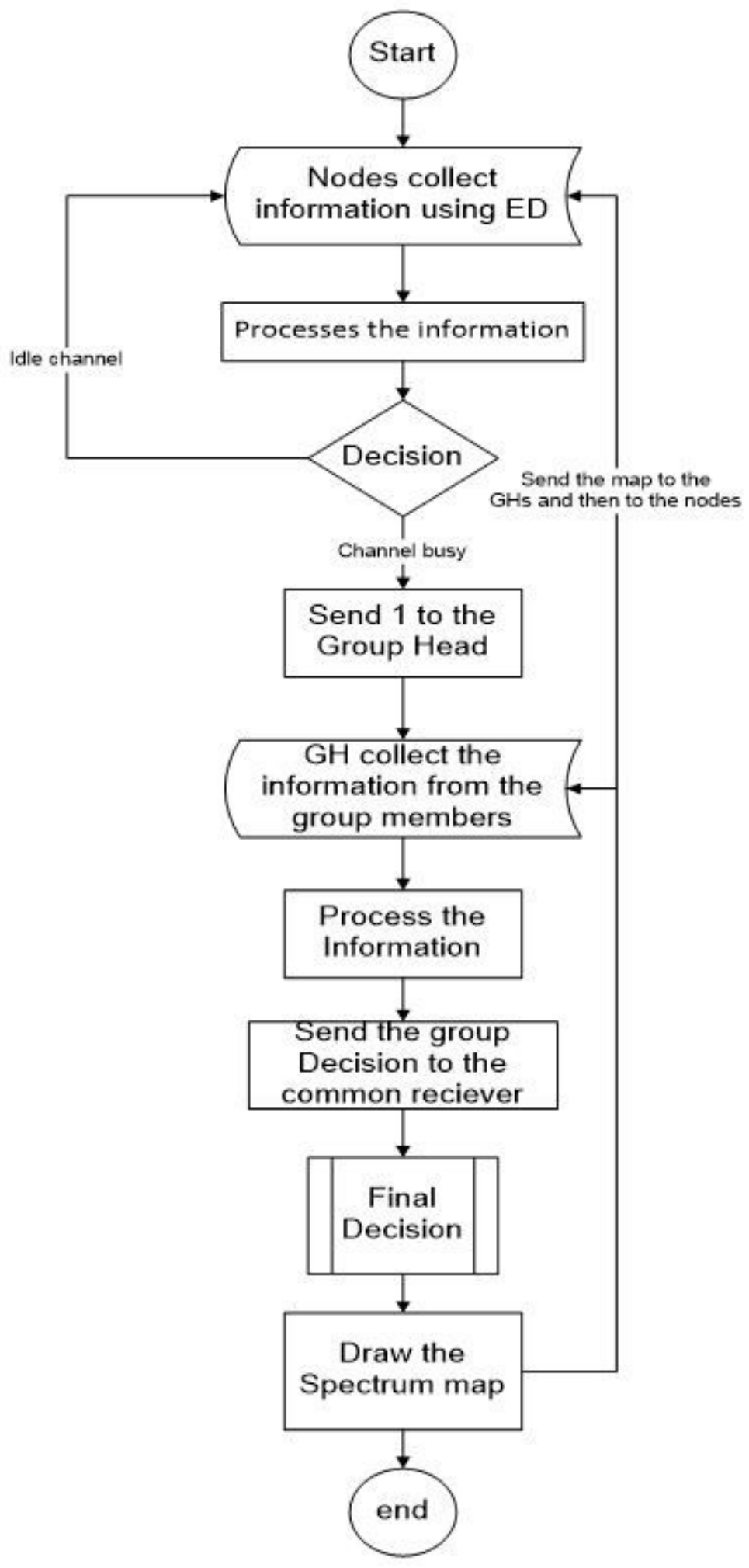

\section{Figure 2}

The decision stages for the distributed cooperative spectrum sensing 


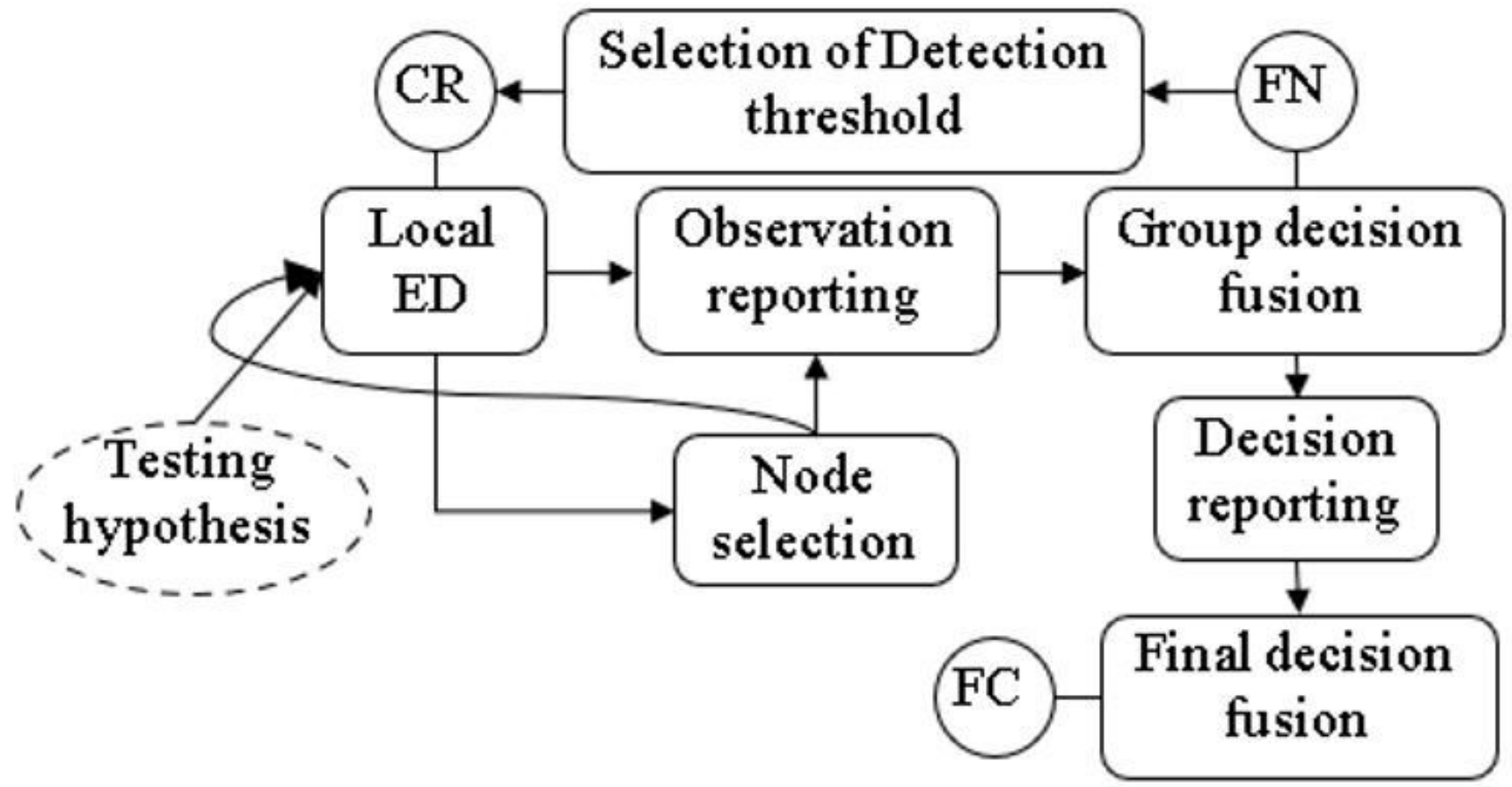

Figure 3

Distributed sensing stages

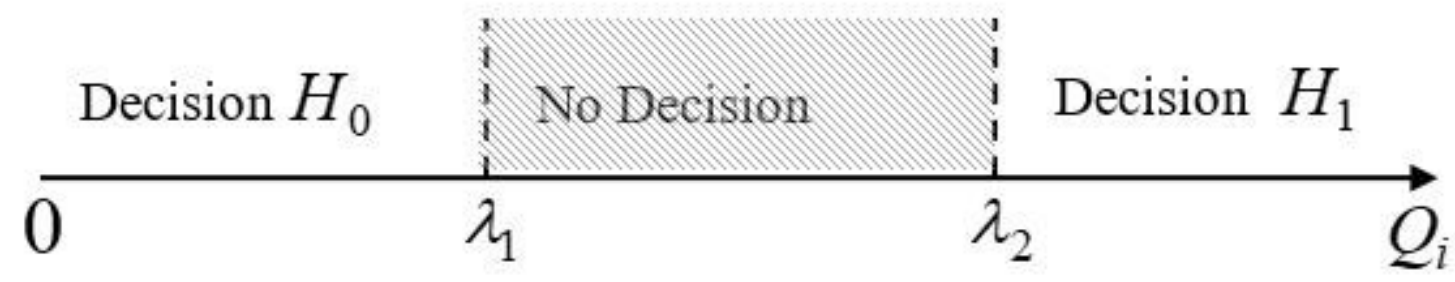

(a)

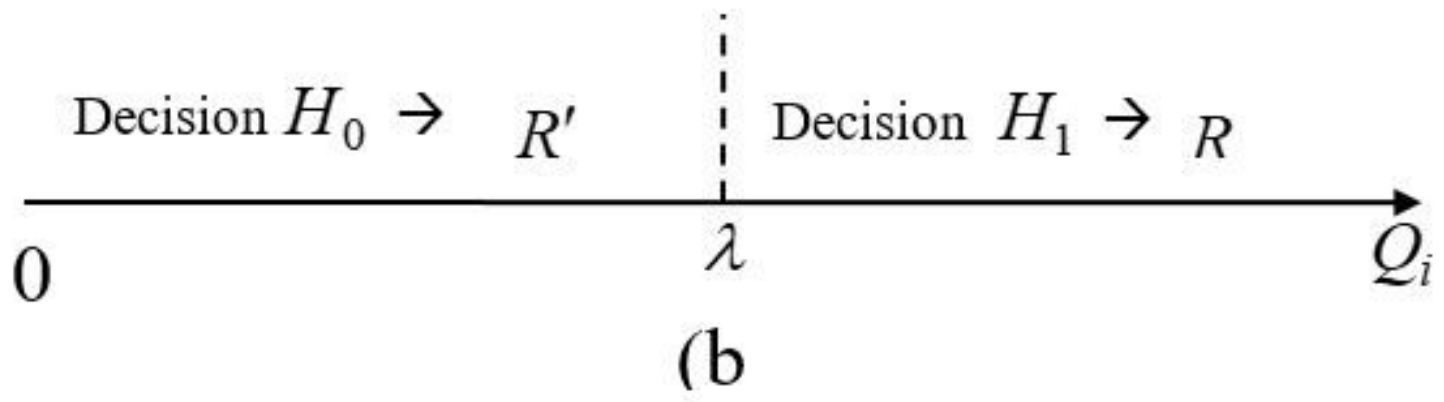

Figure 4 
(a) Cooperative sensing detection technique with two thresholds for local user. (b) Auto-correction technique with single threshold.

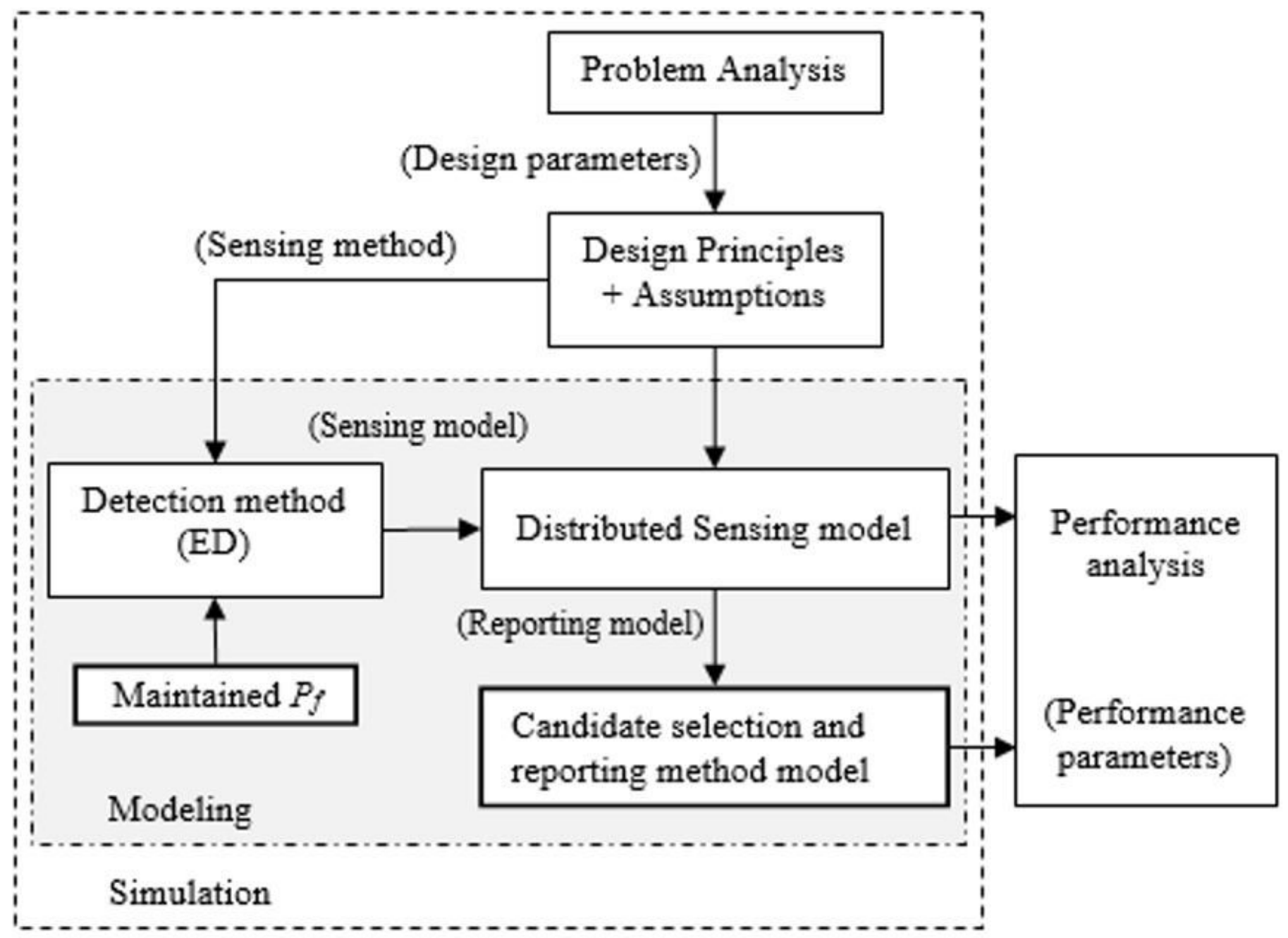

Figure 5

Simulation design and the DCS architecture 


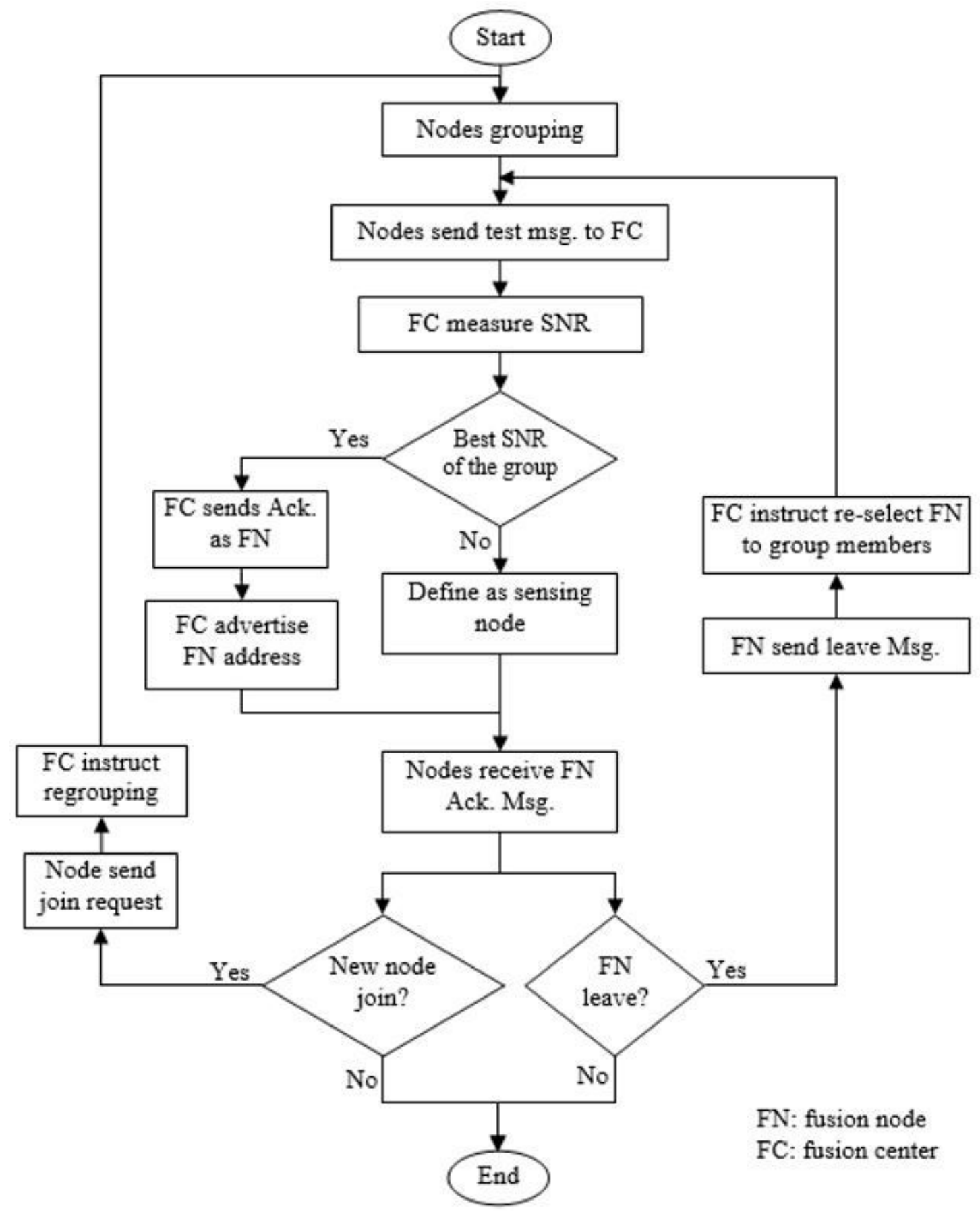

Figure 6

Fusion node selection 


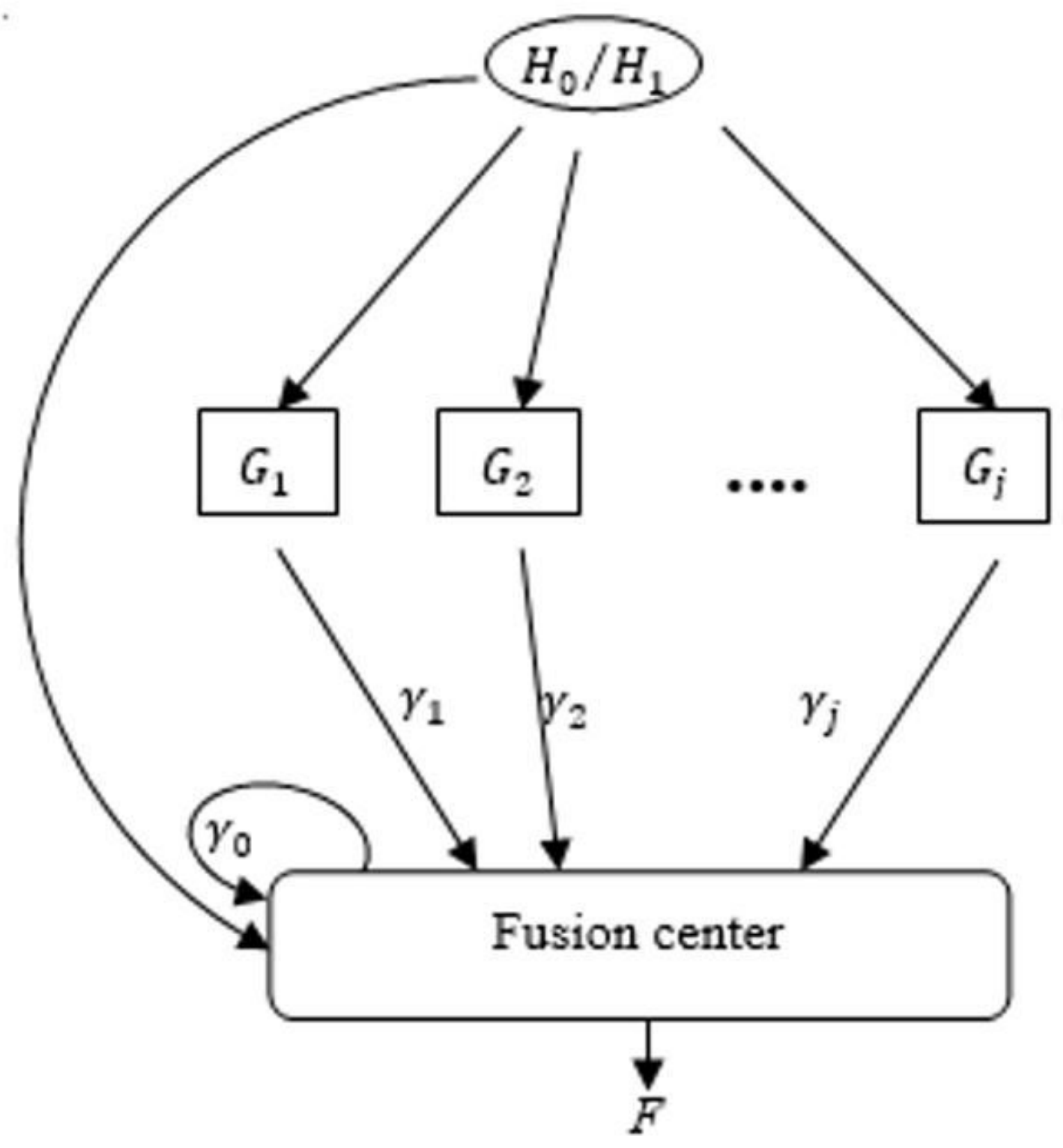

Figure 7

Simulation Architecture 


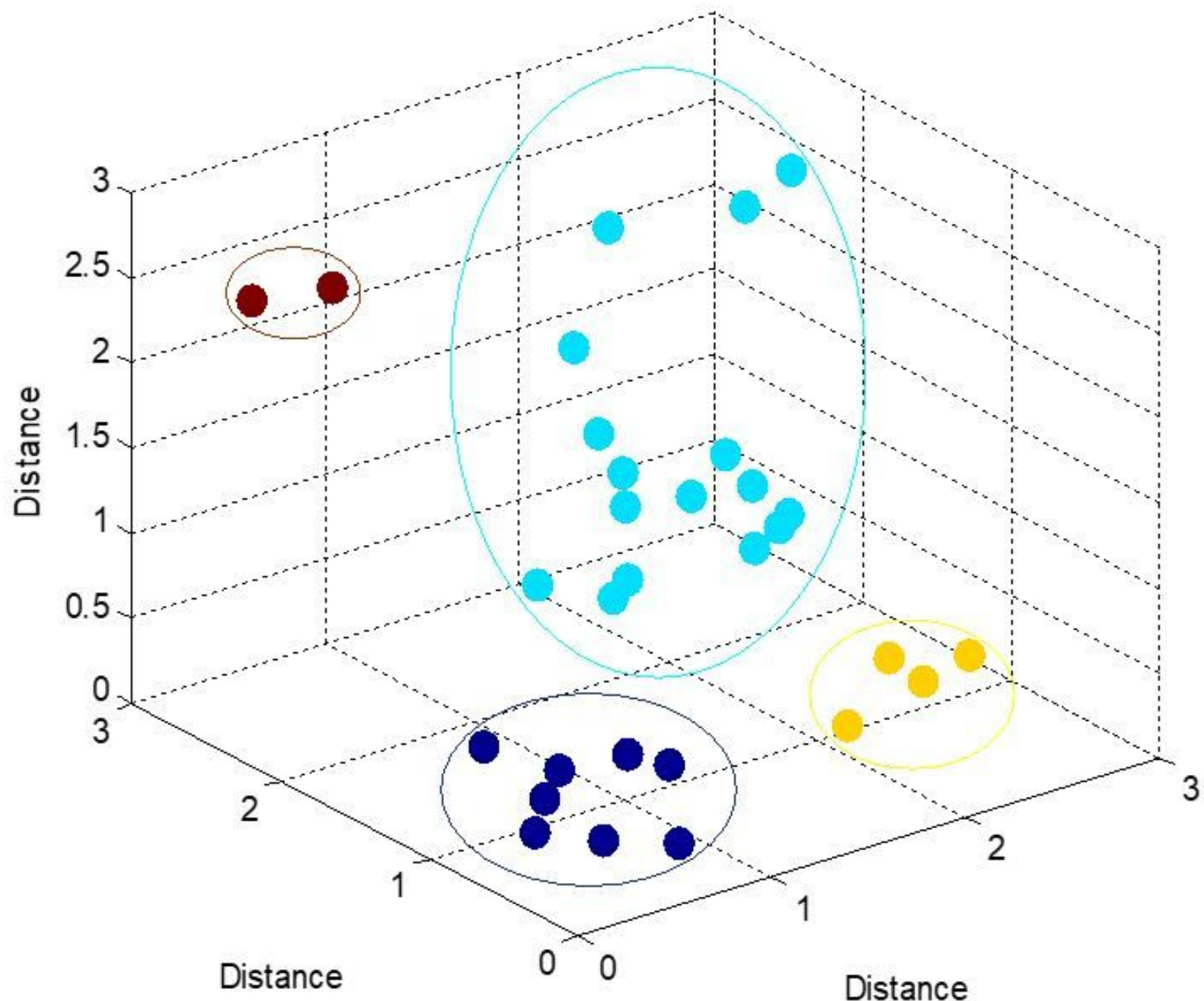

Figure 8

The simulation scatter distribution setup for i.i.d. 30 distributed cognitive radios with different reporting groups 




Figure 9

please see the manuscript file for the full caption 


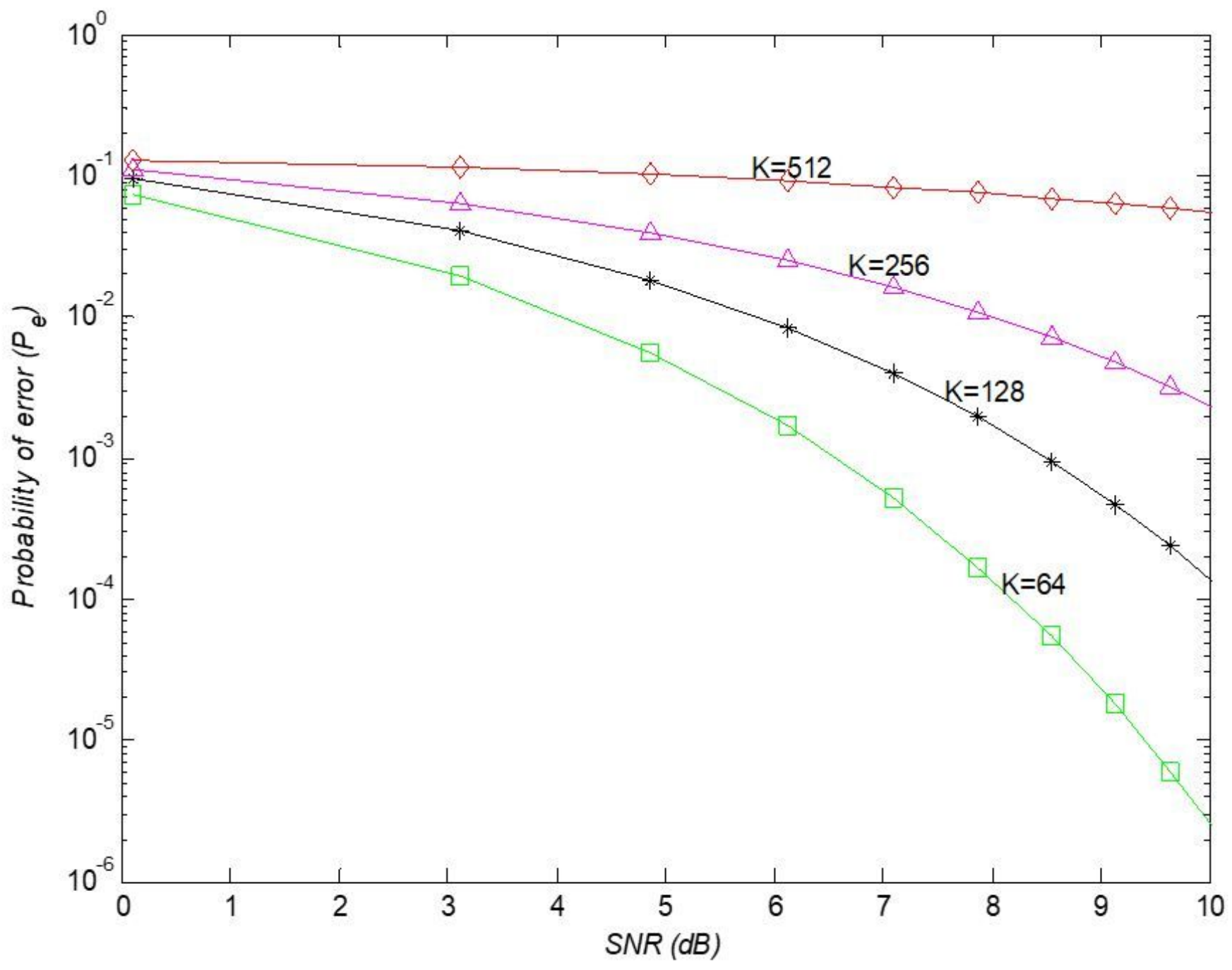

Figure 10

Probability of error for sensing groups allocated with different number of users $(K=64,128,156,512$; $\mathrm{J}=4)$. 


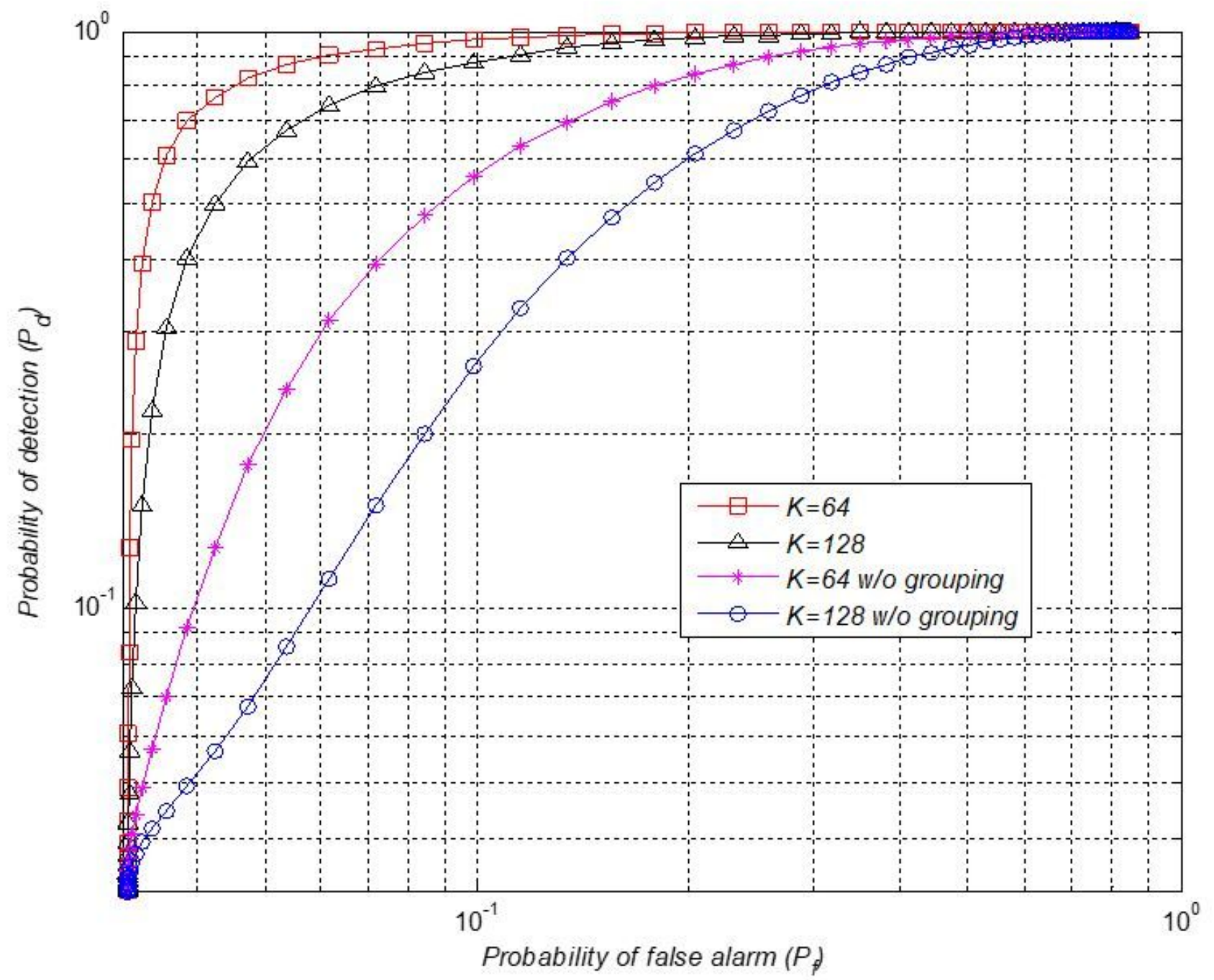

Figure 11

please see the manuscript file for the full caption 


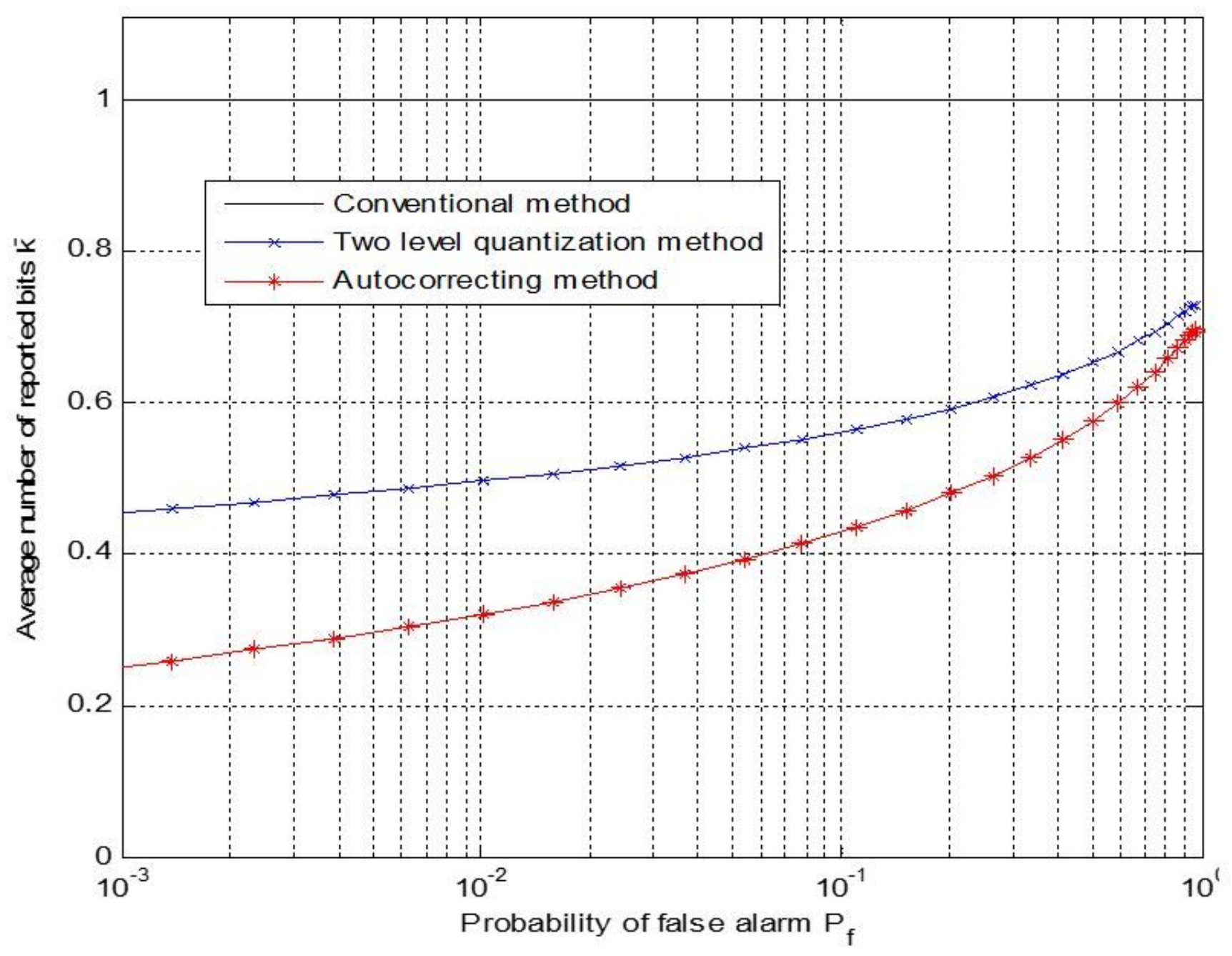

Figure 12

please see the manuscript file for the full caption 


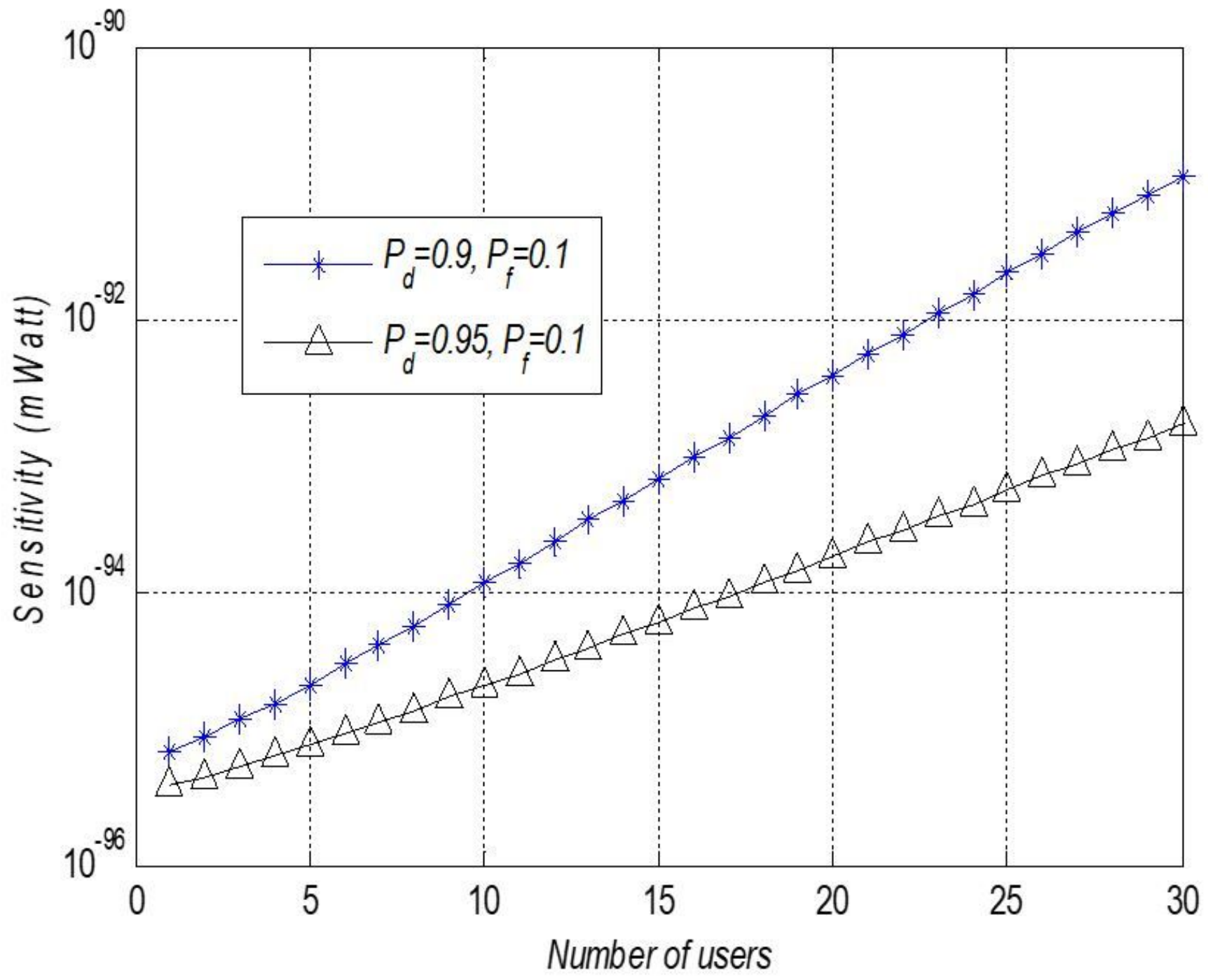

Figure 13

please see the manuscript file for the full caption

Figure 14

Drawback of the distributed cooperative spectrum sensing based OR-rule compared to AND-rule. 